\title{
Entangled photon spectroscopy
}

\author{
Frank Schlawin \\ Clarendon Laboratory, University of Oxford, Parks Road, Oxford OX1 3PU, United Kingdom \\ E-mail: Frank.Schlawin@physics.ox.ac.uk
}

\begin{abstract}
.
This tutorial outlines the theory of nonlinear spectroscopy with quantum light, and in particular with entangled photons. To this end, we briefly review molecular quantum electrodynamics, and discuss the approximations involved. Then we outline the perturbation theory underlying nonlinear spectroscopy. In contrast to the conventional semiclassical theory, our derivation starts from Glauber's photon counting formalism, and naturally includes the semiclassical theory as a special case. Finally, we review previous work, which we sort into work depending on the unusual features of quantum noise, and work relying upon quantum correlations in entangled photons.

This work naturally draws from both quantum optics and chemical physics. Even though it is impossible to provide a comprehensive overview of both fields in one tutorial, this text aims to be self-contained. We refer to specialised reviews, where we cannot provide details. We do not attempt to provide a comprehensive review of all the literature, but rather focus on specific examples intended to elucidate the underlying physics, and merely cite the remaining publications.
\end{abstract}

\section{Contents}

1 Introduction $\quad 2$

1.1 Brief history of quantum spectroscopy . . . . . . . . . . . . 3

2 Quantum electrodynamics $\quad 4$

2.1 Minimal coupling Hamiltonian . . . . . . . . . . . . . . . . . . . . 4

2.2 Dipole approximation . . . . . . . . . . . . . . . . 5

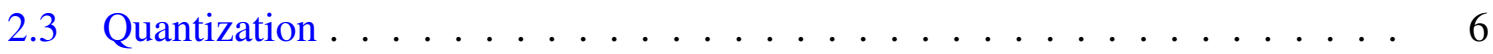

2.3.1 The matter Hamiltonian . . . . . . . . . . . . . . . . 7

2.3.2 The quantized field . . . . . . . . . . . . . . . . . 7

2.4 Molecular interactions \& excitons . . . . . . . . . . . . . . . . 8

2.5 Further approximations in the optical regime . . . . . . . . . . . . . 9

3 Nonlinear spectroscopy $\quad 10$

3.1 Initial state . . . . . . . . . . . . . . . . . . . 11

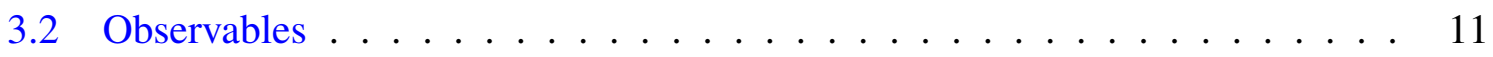

3.3 Diagram construction . . . . . . . . . . . . . . . 13 
4 Photon statistics $\quad 16$



4.2 Light fluctuations in spectroscopy $\ldots \ldots \ldots \ldots \ldots \ldots$

5 Quantum correlations $\quad 18$

5.1 Time-energy entanglement . . . . . . . . . . . . . . . . . 19

5.2 Two-photon induced fluorescence $\ldots \ldots \ldots \ldots \ldots . \ldots . \ldots 22$

5.3 Multidimensional spectroscopic signals . . . . . . . . . . . 25

6 Conclusions 26

\section{Introduction}

Nonlinear laser spectroscopy can reveal valuable information on - inter alia - quasiparticle interactions, or relaxation and transport pathways, which cannot be deduced from the linear response of the sample system. Perhaps most prominently, pump-probe spectroscopy, where a strong laser pulse excites a sample system and a second laser pulse probes the excited transient dynamics, has evolved into an indispensable tool to investigate ultrafast dynamics in complex quantum systems in virtually all fields of condensed matter and chemical physics [95, 94]. In the latter field, multidimensional spectroscopy has further allowed for the investigation of the quantum-mechanical coherence of nonequilibrium processes [1, 2, 3]. The report of wave-like energy transport in the FMO complex in 2007 [11] - followed by similar results in a variety of other samples - has created enormous interest in the possible relevance of quantum-mechanical coherence in photosynthesis [4, 18], in particular, and similar reports in organic solar cells [12] have demonstrated the possible relevance in solar energy harvesting, in general. These findings could be of great importance in possible future quantum technologies [6]. To fully unravel the complex relaxation dynamics in such systems on ultrafast timescales, new methods would be highly desirable, which provide experimentalists with new control knobs to facilitate the determination of model parameters. Apart from classical approaches such as coherent control [7,8], one promising new approach consists in the use of quantum properties of light which is the subject of this tutorial, i.e. quantum spectroscopy.

The research on quantum coherence in energy transport further raised questions regarding the influence of the laser pulse coherence in the experiments and their resulting interpretation [13]. As argued by Brumer and coworkers [14], the observed features directly stem from the excitation by lasers, and bear no relevance for the absorption process under natural light conditions. Naturally, questions regarding the coherence of the light fields are intimately connected to the fields' correlation functions, and thus to Glauber's photon counting theory [16], which form the very basis of quantum optics. So even though this particular discussion is not the subject of this tutorial, the theory for entangled photon spectroscopy provides the natural language, in which such questions can be addressed, and we will comment on these when appropriate.

This tutorial introduces an emerging new strand of nonlinear spectroscopy that aims to 
exploit the peculiar properties of quantum light at low photon fluxes. So when we contrast quantum spectroscopy with "classical" spectroscopy, we imply spectroscopy with coherent states of light with large amplitudes, such that quantum fluctuations can be neglected and a classical treatment of the light fields are sufficient [94]. Ian Walmsley recently identified the defining features of quantum light as "exceptionally low noise and strong correlations" [9], and our tutorial will follow this characterization: After an introduction into molecular quantum electrodynamics in section 2 and the derivation of nonlinear optical signals from a quantum optical perspective in section 3, section 4 discusses work relying on squeezed noise, and the how the reduced noise can be employed to reveal otherwise hidden feature in the sample. Section 5 then focusses on applications that exploit the strong frequency and time correlations, and how these quantum correlations can be exploited to control exciton distributions in complex quantum systems. In contrast to a different recent review [10], this tutorial will try to put emphasis on the background theory, and discuss the derivation thereof in some detail. Naturally, the description of quantum light interaction with a complex quantum system necessitates the application of a number of approximations on different levels of the underlying theory. Providing one consistent account of these approximations will be one of the primary goals of this tutorial. Finally, we conclude in section 6.

\subsection{Brief history of quantum spectroscopy}

Soon after Glauber established the hierarchy of $n$-th order coherence correlation for the characterization of light sources [20], it was pointed out by Mollow [21] that two-photon absorption is proportional to the second-order coherence of the field. In principle, this work already established that photonic correlations could be observed in and influence such transitions. The next notable result was obtained almost thirty years later, when it was predicted theoretically [22, 23] and demonstrated experimentally [26, 27] that the two-photon absorption rate of time-frequency entangled photons scales linearly with the beam intensity. This could enable nonlinear entangled photon spectroscopy at much lower photon fluxes, and thus render it feasible experimentally. At the same time, it also points towards a possible advantage of entangled photon spectroscopy in photosensitive samples, where large photon fluxes are to be avoided. An advantage pertaining specifically to reduced field fluctuations was first demonstrated experimentally in [25], where the squeezed fluctuations of quantum light allowed "an improvement in sensitivity of $3.1 \mathrm{~dB}$ beyond the vacuum-state limit [..] for the detection of Doppler-free resonances in atomic cesium".

The 1990's also witnessed the development of broadband entangled light sources $[28,29]$, which fostered the development of the strong (and possibly quantum) frequency correlations shared between entangled photon pairs [30, 31]. These unusual properties were exploited in a number of imaging and spectroscopic applications, including but not limited to the cancellation of even-order dispersion [36, 37, 38], ghost imaging [32], quantum optical coherence tomography [39, 40], and quantum lithography [41, 42]. Yet it should be noted that in many of these applications, entanglement has shown to be dispensible. For instance, ghost imaging can be carried out with classical light [33], and many two-photon interference effect 
can be simulated with classical light as well $[34,35]$. The proposal most closely connected to this tutorial is entangled photon virtual state spectroscopy [24], which exploits the variation of an inherently nonclassical parameter - the entanglement time (see section 5.1) - to reveal far off-resonant transitions. Unfortunately, this proposed effect depends on the extremely far tails of the photonic frequency spectra, and therefore vanishes after removal of those tails [47]. Therefore, entangled photon spectroscopy still awaits experimental demonstration. Timefrequency entangled photons were first employed as spectroscopic tools in [43]. In this setup, one of the two photons interacts with the sample, while the other is detected in coincidence. The signal is then given by the change in the coincidence count rate. Two-photon absorption of time-frequency entangled photon pairs was first demonstrated in atomic clouds in [44, 45, 46], and, shortly thereafter, in molecules $[48,49,50,51]$. These experiments were more recently followed up by a number of theoretical publications, discussing the application of entangled photon absorption to complex quantum systems [52, 53, 121, 56, 130], the use of entangled photons in Raman spectroscopy [57, 141], and the creation of multidimensional spectroscopic signals with entangled photons $[54,128]$.

\section{Quantum electrodynamics}

In this section, we review the background for the theory of section 3. Starting from a generic minimal coupling Hamiltonian, we discuss dipole expansion, and sketch the derivation of dipole interactions and the exciton picture. A particular focus is paid to the approximations that are made in this derivation. As we will see, those can be critical in the correct determination of coherence properties of the matter or the calculation of off-resonant multiphoton transitions.

More details on the material covered in this section can be found in a number of textbooks on quantum electrodynamics, such as [58, 59,60], open quantum systems [61], or chemical physics [62].

\subsection{Minimal coupling Hamiltonian}

In classical electrodynamics, the electromagnetic fields are described by the scalar potential $\Phi$ and the vector potential $\mathbf{A}$, which interact with point-like particles with charges $e_{\alpha}$ at positions $\mathbf{q}_{\alpha}$. Since the potentials do not fix the observable fields unambiguously, we are free to choose a gauge which eliminates one of the four components of the four-vector $(\Phi, \mathbf{A})$. Non-relativistic light-matter coupling is most conveniently formulated in the Coulomb gauge,

$$
\nabla \cdot \mathbf{A}=0,
$$

which implies that the scalar potential acts instantaneously, satisfying the Poisson equation, and only the vector potential shows retardation. In this case, the Coulomb interaction between point-like charges can be evaluated explicitly, yielding the electrostatic potential

$$
U\left(\mathbf{q}_{1}, \mathbf{q}_{2}\right)=\frac{e_{1} e_{2}}{4 \pi \epsilon_{0}\left|\mathbf{q}_{1}-\mathbf{q}_{2}\right|},
$$


where $\epsilon_{0}$ denotes the vacuum dielectric constant, $e_{i}$ the $i$-th particle's charge and $\mathbf{q}_{i}$ its position. The Coulomb potential can then be subsumed into the matter Hamiltonian, and the Hamiltonian can be solved with the usual methods of nonrelativistic quantum mechanics. Its solution can be used as a starting point for a perturbation expansion. Only the axial modes, described by $\mathbf{A}$, are treated explicitly, and quantized, which is why this gauge is sometimes referred to as the semiclassical gauge. It is important to keep in mind, however, that the semiclassicality does not pertain to the nature of the axial modes, and does not imply the negligence of quantum features of the transverse photons.

The coupling between particles and fields is introduced by the canonical transformation of the particle momenta, $\mathbf{p}_{\alpha} \rightarrow \mathbf{p}_{\alpha}-e_{\alpha} \mathbf{A}\left(\mathbf{q}_{\alpha}\right)$, yielding the minimal coupling Hamiltonian (see. e.g. [59] for a detailed discussion)

$$
H=\sum_{\alpha} \frac{1}{2 m_{\alpha}}\left(\mathbf{p}_{\alpha}-e_{\alpha} \mathbf{A}\left(\mathbf{q}_{\alpha}\right)\right)^{2}+U\left(\mathbf{q}_{1}, \cdots \mathbf{q}_{N}\right)+\frac{1}{2} \int\left(\frac{\Pi^{2}}{\epsilon_{0}}+\epsilon_{0} c^{2}(\nabla \times \mathbf{A})^{2}\right) d^{3} \mathbf{r},(3)
$$

where $\Pi=\Pi=\epsilon_{0} \partial \mathbf{A} / \partial t$ denotes the field momentum, $U$ the electrostatic interactions given by Eq. (2) and $c$ the vacuum speed of light. We employ SI units. It contains two coupling terms between field and matter: the paramagnetic interaction $\sum_{\alpha} e_{\alpha} \mathbf{p}_{\alpha} \mathbf{A}\left(\mathbf{q}_{\alpha}\right) / m_{\alpha}$, and the diamagnetic interaction $\sum_{\alpha} e_{\alpha}^{2} \mathbf{A}^{2}\left(\mathbf{q}_{\alpha}\right) /\left(2 m_{\alpha}\right)$.

In principle, this Hamiltonian could be used as a starting point for the description of spectroscopic signals. However, in the description of molecular signals, it has several important drawbacks. For one, the electrostatic interaction $U$ contains both intramolecular as well as intermolecular terms,

$$
U\left(\mathbf{q}_{1}, \cdots \mathbf{q}_{N}\right)=\sum_{n=1}^{M} U_{\text {intra }}\left(\mathbf{q}_{n_{1}}, \ldots \mathbf{q}_{n_{M}}\right)+U_{\text {inter }}\left(\mathbf{q}_{1}, \ldots \mathbf{q}_{N}\right)
$$

where the summation in the first term runs over the $M$ particles that form molecule $n$. It would be much preferable to work in a gauge, in which the electrostatic interaction only describes the interaction within each molecule, while intermolecular interactions are described by the transverse modes of the field. And second, the minimal coupling Hamiltonian does not exploit the distinct length scales of the problem: Optical wavelengths are orders of magnitude larger than typical atomic or molecular distances, such that the light field can be approximated as constant (or nearly constant) on molecular scales. These considerations naturally lead to the dipole approximation, which will be discussed next.

\subsection{Dipole approximation}

The equations of motion are naturally invariant under unitary transformations acting on both the Hamiltonian and the eigenfunctions. In particular, we consider the unitary transformation

$$
U=\exp \left[-\frac{i}{\hbar} \int \mathbf{P} \cdot \mathbf{A} d^{3} r\right]
$$

where $\mathbf{P}$ is the polarization field, which we can be expanded in multipole moments of the charge distribution [59]. This transformation changes the field momentum in the 
Hamiltonain (3) from the electric field to the displacement field. Here, we only state the final result to leading order - the dipolar approximation, which reads

$$
\begin{aligned}
H_{d i p} & =U H U^{\dagger} \\
& =\sum_{n} H_{n}+\frac{\epsilon_{0}}{2} \int\left(\mathbf{E}^{2}+c^{2} \mathbf{B}^{2}\right) d^{3} \mathbf{r}-\sum_{n} \hat{\mu}_{n} \mathbf{E}\left(\mathbf{r}_{n}\right),
\end{aligned}
$$

where

$$
H_{n}=\sum_{\alpha=1}^{M} \frac{1}{2 m_{\alpha}} \mathbf{p}_{\alpha}^{2}+U_{\text {intra }}
$$

is the $n$-th molecular Hamiltonian, and the second term denotes the free space field Hamiltonian. The molecules now couple to the light field with their dipole moments,

$$
\hat{\mu}_{n}=-e \sum_{\alpha}\left(\mathbf{q}_{\alpha}-\mathbf{r}_{n}\right)
$$

which couples to the electric field operator $\mathbf{E} \neq$. Notably, the electrostatic intermolecular coupling $U_{\text {inter }}$ of Eq. (4) has been deleted, and the molecules can only interact via the exchange of transverse photons. This interaction will be discussed in section 2.4 later on. We have further neglected the polarization self-energy in the dipolar Hamiltonian (6), since it is not relevant for this tutorial. It should however be taken into account, e.g., for the calculation of Lamb shifts.

Whenever the fields vary appreciably over the size of the sample, the dipole approximation becomes questionable, and higher-order moments of the fields need to be taken into account. In such cases, the minimal coupling Hamiltonian may be more suitable to tackle the problem. Notably, this can be the case in the quantum electrodynamics of condensed matter systems [63, 64, 65], or in nanofield electrodynamics [66]. Finally it is worth noting that even though the dipole approximation works excellently in most cases of interest in molecular systems, it does constitute an approximation, and deviations may be observable [69].

\subsection{Quantization}

The dipole Hamiltonian (6) is quantized by imposing the usual canonical commutation relations [59],

$$
\begin{aligned}
& {\left[p_{i \alpha}, q_{j \alpha}\right]=i \hbar \delta_{i j},} \\
& {\left[A_{i}(\mathbf{r}), \mathbf{E}_{j}\left(\mathbf{r}^{\prime}\right)\right]=i \hbar \delta_{i j}^{\perp}\left(\mathbf{r}-\mathbf{r}^{\prime}\right) .}
\end{aligned}
$$

where $\delta^{\perp}$ denotes the transverse delta-function. In free space, a natural starting point to treat the full system consists in finding solutions of the uncoupled systems. The interaction Hamiltonian will then be treated as a perturbation.

\$ Strictly speaking, as mentioned above, the field in Eq. (6) is the displacement field $\mathbf{d} / \epsilon_{0}$ [59]. In free space (outside of the molecular charge distribution), which we shall be concerned with, the displacement field coincides with the electric field, and to avoid clutter in the notation, we only consider $\mathbf{E}$, and focus on the field quantization in free space in the following. 
2.3.1. The matter Hamiltonian The diagonalization of the molecular Hamiltonian $H_{n}$ in Eq. (6) yields its spectral decomposition

$$
H_{n}=\sum_{k} \epsilon_{n k}|n k\rangle\langle n k|,
$$

which constitutes the so-called "local" basis in photosynthetic complexes. The dipole operator couples these states with the matrix elements,

$$
\mu_{n, k k^{\prime}}=\left\langle n k^{\prime}|\hat{\mu}| n k\right\rangle \text {. }
$$

2.3.2. The quantized field The electromagnetic field Hamiltonian can be written as a collection of harmonic oscillators,

$$
H_{\text {field }}=\sum_{\mathbf{k}, \lambda} a_{(\lambda)}^{\dagger}(\mathbf{k}) a_{(\lambda)}(\mathbf{k}),
$$

where we have already subtracted the zero-point energy, and introduced the photon creation and annihilation operators, $a_{(\lambda)}^{\dagger}(\mathbf{k})$ and $a_{(\lambda)}(\mathbf{k})$, which create or destroy a photon with momentum $\mathbf{k}$ and polarization $\lambda$. They satisfy the usual bosonic commutation relation $\left[a_{(\lambda)}(\mathbf{k}), a_{\left(\lambda^{\prime}\right)}^{\dagger}\left(\mathbf{k}^{\prime}\right)\right]=\delta_{\lambda, \lambda^{\prime}} \delta_{\mathbf{k}, \mathbf{k}^{\prime}}$. The electric field operator is expanded in this basis as

$$
\mathbf{E}(\mathbf{r}, t)=i \sum_{\mathbf{k}, \lambda} \sqrt{\frac{\hbar \omega_{\mathbf{k}}}{2 \epsilon_{0} V}} \hat{e}_{\mathbf{k}}^{(\lambda)} a_{(\lambda)}(\mathbf{k}) e^{i \mathbf{k r}} e^{-i \omega_{k} t}+\text { h.c. }
$$

where $V$ denotes the quantization volume.

The box quantization introduced above can be found in most textbooks, and it can be used to calculate spectroscopic signals. For our purposes, another representation is more convenient. We typically focus on a situation as sketched in Fig. 1, where incident light fields with well-defined propagation directions are impinging on a localised sample. Thus, to describe one such field, we only want to take into account the field modes along this propagation direction. We can further make life easier by changing to a continuous frequency distribution, $\sum_{k} \rightarrow 1 / \Delta \omega \int d \omega$, and obtain the continuous field representation in the interaction picture with respect to the field Hamiltonian $H_{\text {field }}[70,71]$

$$
\mathbf{E}(z, t)=\mathbf{E}^{\dagger}(z, t)+\mathbf{E}(z, t),
$$

with the positive frequency component

$$
\mathbf{E}(z, t)=i \hat{e} \int_{0}^{\infty} d \omega \sqrt{\frac{\hbar \omega}{4 \pi \epsilon_{0} c A}} e^{i \omega(z / c-t)} a(\omega),
$$

$E^{\dagger}$ its hermitian conjugate, and the corresponding permutation relation $\left[a(\omega), a^{\dagger}\left(\omega^{\prime}\right)\right]=$ $\delta\left(\omega-\omega^{\prime}\right)$. Here, $z$ denotes the spatial coordinate along the field's propagation direction, $\hat{e}$ a polarization vector, and $A$ the quantization area perpendicular to the propagation direction. 


\subsection{Molecular interactions $\mathcal{E}$ excitons}

The dipole approximation in Eq. (6) has eliminated the direct electrostatic coupling $U_{\text {inter }}$ between molecules, and it seems that we have separated the dynamics into those of noninteracting molecules. This is not true, of course, since the molecules can still interact via the exchange of virtual photons. This retarded dipole interaction can be calculated in second-order perturbation theory [72]. We then obtain the matrix element for the resonant transfer of an excitation between molecules $n$ and $m$,

$$
\begin{aligned}
& J=\frac{3 \sqrt{\gamma_{n} \gamma_{m}}}{2}\left\{-\left[\hat{\mu}_{n} \cdot \hat{\mu}_{m}-\left(\hat{\mu}_{n} \cdot \hat{\mathbf{r}}_{n m}\right)\left(\hat{\mu}_{m} \cdot \hat{\mathbf{r}}_{n m}\right)\right] \frac{\cos \xi_{n m}}{\xi_{n m}}\right. \\
& \left.+\left[\hat{\mu}_{n} \cdot \hat{\mu}_{m}-3\left(\hat{\mu}_{1} \cdot \hat{\mathbf{r}}_{n m}\right)\left(\hat{\mu}_{m} \cdot \hat{\mathbf{r}}_{n m}\right)\right]\left(\frac{\sin \xi_{n m}}{\xi_{n m}^{2}}+\frac{\cos \xi_{n m}}{\xi_{n m}^{3}}\right)\right\},
\end{aligned}
$$

where $\xi_{n m} \equiv \omega_{0} r_{n m} / c$ and $\gamma_{n}=\mu_{n}^{2} \omega_{n}^{3} / 6 \pi \epsilon_{0} \hbar c^{3}$. Here, $\omega_{n}=\left(\epsilon_{n}-\epsilon_{n 0}\right) / \hbar$ is the $n$-th molecular transition frequency, $\omega_{0}=\left(\omega_{n}+\omega_{m}\right) / 2$, and $\hat{r}_{n m}$ a unit vector connecting molecules $n$ and $m$.

Within molecular aggregates, this dipolar interaction couples the eigenstates in Eq. (11) into new, delocalised eigenstates of an effective Hamiltonian including Eq. (17). Excitations in these new eigenstates can be treated as quasiparticles, called excitons. Given the spectral decomposition of the molecular Hamiltonian (11), we can define an excitation annihilation operator as [92]

$$
b_{n} \equiv|n 0\rangle\langle n 1|+\sqrt{2}| n 1\rangle\langle n 2|+\ldots .
$$

The resulting Hamiltonian of one such aggregate can be written as

$$
H_{\text {agg }}=\sum_{n} \epsilon_{n} b_{n}^{\dagger} b_{n}+\frac{1}{2} \sum_{n \neq m} J_{n m} b_{n}^{\dagger} b_{m}+\sum_{m, n, k, l} U_{m n k l} b_{n}^{\dagger} b_{m}^{\dagger} b_{k} b_{l},
$$

where the excitation creation and annihilation operators $b_{n}$ and $b_{n}^{\dagger}$ obey nearly bosonic commutation relations,

$$
\left[b_{n}, b_{m}^{\dagger}\right]=\delta_{n m}\left(1-\frac{3}{2} b_{m}^{\dagger 2} b_{m}^{2}\right) .
$$

Due to the couplings $J_{n m}$, spectroscopic resonances are shifted energetically with respect to the bare molecular transitions, and it is the excitons that one detects in spectroscopic measurements of molecular aggregates. When the molecules are very closely packed, higher moments of their charge distributions can become important, and deviations from the dipole approximation can be observed below $\sim 15 \AA$ in large photosynthetic complexes [67, 68].

We discussed this derivation in detail because it is important to keep in mind that once we adopt an excitonic Hamiltonian for the sample system, quantum light effects have already been taken into account. As we will see later in section 3.3, these naturally show up in the derivation of nonlinear spectroscopic signals in the quantum regime as well. Thus, great care has to be taken in the discussion of quantum light effects in nonlinear spectroscopy $[75,76,77,78]$ to avoid the double counting of these photon exchange processes. Similarly, in writing the effective interaction (17), we have neglected collective decay processes. These give rise to the spontaneous creation of excitonic coherence [73]. So even if these processes can be neglected 
in the calculation of Förster transfer rates, this need not be the case in quantum coherent transport in molecular aggregates.

Diagonalising the Hamiltonian (19), we obtain

$$
H_{a g g}=\hbar \sum_{e} \omega_{e}|e\rangle\left\langle e\left|+\hbar \sum_{f} \omega_{f}\right| f\right\rangle\langle f|+\ldots
$$

where we denote the single-exciton states $|e\rangle$ and the two-exciton states $|f\rangle$. In four-wave mixing signals, as we will discuss in this tutorial, these two manifold are sufficient to calculate the optical response of the system. While we can formally write down the spectral decomposition (21), let us note that, in practice, its calculation poses a formidable problem requiring both intuition and heavy numerics. In fact, the reliable determination of on-site energies $\epsilon_{n k}$ in Eq. (11) is one of main obstacles in our understanding of energy transport in photosynthetic complexes, and need to be obtained from fitting to optical signals [74].

\subsection{Further approximations in the optical regime}

The dipolar interaction in Eq. (6) can be further simplified. It contains (nearly) energyconserving terms, where an excitation of the matter is accompanied by the destruction of a photon, and vice versa, as well as non-energy-conserving terms, where the excitation is accompanied by a photon creation. Intuitively, it is clear that as long as the coupling between light fields and matter is weak, the former terms will dominate the dynamics. To extract those, we write the dipole operator in the interaction picture [see Eq. (28) below] as

$$
\hat{\mu}_{n}(t)=\mathbf{V}_{n}(t)+\mathbf{V}_{n}^{\dagger}(t)
$$

where $V(t)$ is its positive-frequency component,

$$
\mathbf{V}_{n}(t)=\mu_{n} b_{n} e^{-i \omega_{n} t},
$$

and $\mathbf{V}_{n}^{\dagger}$ the hermitian conjugate. Only retaining terms that oscillate as $\omega-\omega_{n}$, we arrive at the rotating wave approximation Hamiltonian

$$
H_{\text {int }}=\sum_{n} \mathbf{V}_{n}(t) \mathbf{E}^{\dagger}(t)+\mathbf{V}_{n}^{\dagger}(t) \mathbf{E}(t)
$$

When we consider optical fields, the centre frequency $\omega_{0}$ of the involved fields is typically much larger than the fields' bandwidths $\sigma$. In this case, the field normalisation in Eq. (16) hardly changes over the relevant frequency window, we can write approximately $\left(\hbar \omega /\left(4 \pi \epsilon_{0} c A\right)\right)^{1 / 2} \simeq\left(\hbar \omega_{0} /\left(4 \pi \epsilon_{0} c A\right)\right)^{1 / 2}$, and move the term outside of the frequency integral in Eq. (16). Hence, the electric field is then proportional to the annihilation operator,

$$
\mathbf{E}(t)=i \hat{e} \sqrt{\frac{\hbar \omega_{0}}{4 \pi \epsilon_{0} c A}} a(t) .
$$

This is called the slowly varying envelope approximation. The same reasoning allows us to extend the lower integration boundary in Eq. (16) to $-\infty$, thus turning many frequency integrations into Fourier integrals that can be solved analytically. It is, however, important to keep in mind that this represents an approximation which may fail in the description of far off-resonant transitions, as is the case, for instance, in entangled virtual state spectroscopy [24], as well as in multi-photon and strong field problems. 


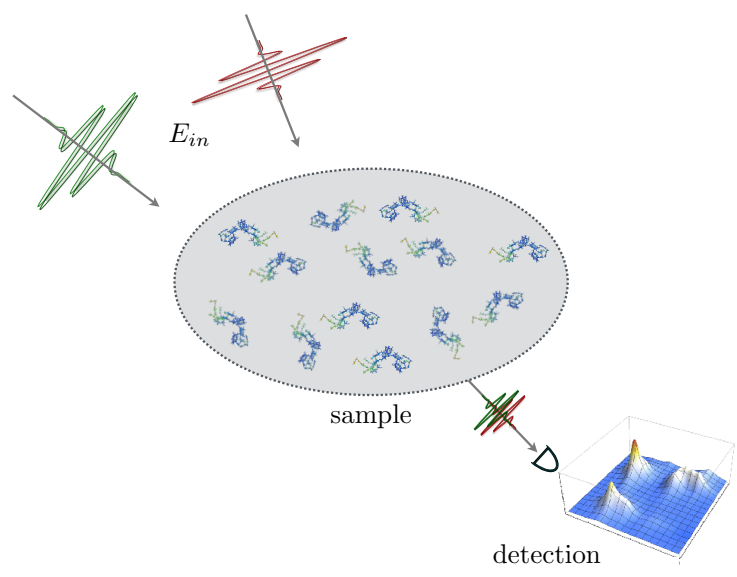

Figure 1. The situation considered in this tutorial: A sample consisting of $N$ molecules (or molecular aggregates) is excited by incoming fields $E_{\text {in }}$ with well-defined propagation directions, described by wavevectors $\mathbf{k}_{1}$ and $\mathbf{k}_{2}$. The signal is created through the detection of either transmitted fields or the induced fluorescence.

\section{Nonlinear spectroscopy}

The general setup we aim to describe is sketched in Fig. 1: A collection of $N$ molecules (or molecular aggregates) is excited by incoming quantum light pulses, and the signal is taken by some form of photon counting experiment after their interaction with the sample. In the absence of dissipation, the detected signal is composed of the coherent superposition of contributions from each molecule, but fluctuations in the surrounding media etc. will quickly destroy phase coherence between distinct emitters. In this tutorial, we shall focus on nonlinear optical signals stemming from individual molecules (or molecular aggregates), and neglect collective effects such as Dicke superradiance [76] and others [77, 78], which can become important at large densities. Therefore, we only propagate the single-molecule density matrix (as opposed to the $N$-molecule matrix necessary to describe collective signals). Such an approach typically requires the rotational averaging over the arbitrary orientation of the molecules with respect to the incoming fields. This procedure is explained in [79, 92]. For simplicity, we assume this average to be accounted for by a renormalization of the dipole strengths§, and write the dipole Hamiltonian (24) as

$$
H_{\text {int }}(t)=V(t) E^{\dagger}(t)+V^{\dagger}(t) E(t),
$$

which will be the basis of the following perturbation expansion.

Given the interaction Hamiltonian (26), we can immediately write down the field-matter density matrix at time $t$ as a Dyson series in the interaction picture with respect to the matter Hamiltonian $H_{0}$ and the field Hamiltonian $H_{f}$,

$$
\varrho(t)=\mathcal{T} \exp \left[-\frac{i}{\hbar} \int_{t_{0}}^{t} d \tau H_{\text {int },-}(\tau)\right] \varrho\left(t_{0}\right),
$$

$\S$ This approach is not fully justified in realistic models, since the fixed relative dipole angle in two-photon transitions is neglected. But since we only consider toy models in following, we shall not concern ourselves with the technicalities of the rotational average. 
with the interaction picture Hamiltonian

$$
H_{\text {int }}(\tau) \equiv e^{i\left(H_{0}+H_{f}\right) \tau / \hbar} H_{\text {int }} e^{-i\left(H_{0}+H_{f}\right) \tau / \hbar} .
$$

We have further defined the Liouville space superoperator $H_{\text {int },-} X \equiv H_{\text {int }} X-X H_{\text {int }}$, and introduced the time-ordering operator $\mathcal{T}$, defined by

$$
\mathcal{T} A(t) B\left(t^{\prime}\right) \equiv \Theta\left(t-t^{\prime}\right) A(t) B\left(t^{\prime}\right)+\Theta\left(t^{\prime}-t\right) B\left(t^{\prime}\right) A(t) .
$$

We will obtain nonlinear optical signals in the following by expanding the time evolution operator in Eq. (27) to the desired order in the number of matter-field interactions. This will result in convolutions of field and matter correlation functions which are evaluated with respect to their respective initial states. We therefore define $\langle\ldots\rangle_{0}$ the expectation value with respect to the initial state $\varrho\left(t_{0}\right)$, in contrast to the expectation value $\langle\ldots\rangle$ of the final state $\varrho(t)$, which contains all orders of the interaction Hamiltonian.

\subsection{Initial state}

In this tutorial, we only consider factorising initial conditions, with the matter system in its ground state $|g\rangle$, i.e.

$$
\varrho\left(t_{0}\right)=\left|g\left(t_{0}\right)\right\rangle\left\langle g\left(t_{0}\right)\right| \otimes \rho_{\text {field }}\left(t_{0}\right) .
$$

This is a very intuitive assumption for the interaction of optical light pulses with a sample system prior to their interaction, when they clearly factorize. Furthermore, in the optical regime thermal occupation of excited electronic states may be safely neglected, since the corresponding temperature amounts to several thousand Kelvin: For instance, a transition at $680 \mathrm{~nm}$ corresponds to a temperature of approximately $4300 \mathrm{~K}$. Of course, this reasoning does not apply to the vibrational degrees of freedom. We assume that the coupling to electronic states is weak, and may be captured by a suitable master equation. When the sample system features strong coupling to vibrations [82], which could underlie the extraordinary quantum efficiency of certain photosynthetic complexes [83], this assumption fails, and the ground state would need to be replaced by a suitable thermal state of the coupled system. This could lead to new contributions to the nonlinear signals we will derive in the following.

\subsection{Observables}

According to Glauber's theory of photodetection, which we will briefly sketch in section 4, field measurements correspond to the normally-ordered field operators. We thus consider as observable an arbitrary function of normal-ordered annihilation and creation operators,

$$
O(t)=f\left(\left\{a_{i}^{\dagger}(t)\right\},\left\{a_{i}(t)\right\}\right),
$$

where the subscripts distinguish the fields in different detectors. The expectation value reads, using Eq. (27),

$$
\begin{aligned}
\langle O\rangle & =\int_{-\infty}^{\infty} d t \operatorname{tr}\left\{\mathcal{T} O(t) \exp \left[-\frac{i}{\hbar} \int_{t_{0}}^{t} d \tau H_{\text {int, },}(\tau)\right] \varrho\left(t_{0}\right)\right\} \\
& =\int_{-\infty}^{\infty} d t\left\langle\mathcal{T} O(t) \exp \left[-\frac{i}{\hbar} \int_{t_{0}}^{t} d \tau H_{\text {int },-}(\tau)\right]\right\rangle_{0} .
\end{aligned}
$$


The expectation value is now taken with respect to the initial state $\varrho\left(t_{0}\right)$. We integrated over the time $t$, at which the signal is produced, since typically the time resolution of photo detectors is much lower than the optical time scales of broadband laser pulses. This definition is unsuited for $\mathrm{cw}$ excitation, as the time integration of a constant value diverges. Hence, in this situation, the signal should be replaced by an appropriate average, i.e.

$$
\langle O\rangle_{c w}=\lim _{T \rightarrow \infty} \frac{1}{2 T} \int_{-T}^{T} d t\left\langle\mathcal{T} O(t) \exp \left[-\frac{i}{\hbar} \int_{t_{0}}^{t} d \tau H_{\text {int, }}(\tau)\right]\right\rangle_{0} .
$$

Information on the sample system is encoded in the changes of the expectation value induced by the interaction with the sample. Thus, without loss of generality, we set the expectation value in the absence of the sample to zero, i.e. $\langle O\rangle_{0}=0$. The change due to the interaction with the sample then defines the signal $S(\Gamma)$, where $\Gamma$ shall denote the set of control parameters of the sample, such as frequencies, time delays, or properties of the quantum state of the initial light field.

Using the identity [87]

$$
\left(E^{\dagger} V\right)_{-}(t)=E_{+}^{\dagger}(t) V_{-}(t)+E_{-}^{\dagger}(t) V_{+}(t),
$$

where we define the anti-commutator superoperator $V_{+} X \equiv=(V X+X V) / 2 \|$, we can expand Eq. (33) to first order, and obtain

$$
S(\Gamma)=-\frac{i}{\hbar} \int_{-\infty}^{\infty} d t \int_{t_{0}}^{t} d \tau\left\{\left\langle[O(t), E(\tau)]_{+} V_{+}^{\dagger}(\tau)\right\rangle+\left\langle\left[O(t), E^{\dagger}(\tau)\right]_{+} V_{+}(\tau)\right\rangle\right\} .
$$

This equation allows to calculate the optical signals for arbitrary observables, such as photon counting or quadrature measurements. In the following, we explicitly evaluate two examples - absorption and fluorescence. For further examples and additional information, we refer to chapter 2 of [93].

Absorption An absorption measurement records the depletion of an optical field through its interaction with a sample. The corresponding observable is given by the photon number operator,

$$
\mathcal{O}_{a b s}(t)=a^{\dagger}(t) a(t)
$$

Eq. (36) then yields

$$
S_{\text {int }}(\Gamma)=\frac{2}{\hbar} \mathfrak{J} \int_{-\infty}^{\infty} d t\left\langle E_{+}^{\dagger}(t) V_{+}(t)\right\rangle .
$$

This result can also be obtained without reference to photon counting by evaluating the energy change of the electromagnetic field [88]. If we replace the field operator $E_{+}^{\dagger}$ in Eq. (38) by a classical field amplitude, we obtain $S_{\text {int }} \sim \mathfrak{J}\left\langle V_{+}(t)\right\rangle$. This is just the semiclassical result, where the expectation value of the dipole operator acts as the source for the polarization field [94]. Naturally, it emerges as the coherent state limit of the current approachף.

\| The factor $1 / 2$ is introduced for normalization.

II Strictly speaking, we also need to neglect quantum fluctuations corresponding to field commutators in the correlation functions (see Eq. (41) below), so we also require large coherent field amplitudes. 
Fluorescence signals Just like absorption, Eq. (38), fluorescence signals measure the intensity of the light field, corresponding to the observable (37). In contrast to absorption, however, we here consider the spontaneous decay of a molecular excitation into the vacuum. Therefore, even though Eq. (38) remains formally correct, we can further simplify the signal by tracing out the vacuum modes. We obtain [93]

$$
S_{\text {Fluor }}(\Gamma)=\frac{2 \pi\left|\mathcal{E}_{0}\right|^{2}}{\hbar^{2}} \int d t\left\langle V_{L}(t) V_{R}^{\dagger}(t)\right\rangle,
$$

where $\mathcal{E}_{0}=\left(\hbar \omega_{0} /\left(4 \pi \epsilon_{0} c A_{0}\right)\right)^{1 / 2}$ denotes the vacuum field normalisation [see Eq. (16)]. We have further defined the left- and right-superoperators $V_{L}=V_{+}+V_{-} / 2$ and $V_{R}^{\dagger}=V_{+}-V_{-} / 2$, i.e. $V_{L} X=V X$ and $V_{R} X=X V$. This simply means that a fluorescence signal is associated with the de-excitation of both sides of the density matrix. An excited state population is destroyed, as opposed to a coherence in the absorption signal (38).

\subsection{Diagram construction}

We now have all the ingredients in place: the final density matrix (27) with the time evolution operator, and the observables, which create the signal (36). The final step of the theory consists in the construction of the optical signals. Expanding the exponential of the Dyson series in Eq. (27) to $N$-th order, and inserting the result in Eq. (36), we obtain expressions of the form

$$
\begin{aligned}
& S^{(N)}(\Gamma)=-\frac{i}{\hbar}\left(-\frac{i}{\hbar}\right)^{N} \int_{-\infty}^{\infty} d t \int_{t_{0}}^{t} d \tau \int_{t_{0}}^{\tau} d \tau_{N} \ldots \int_{t_{0}}^{\tau_{2}} d \tau_{1} \\
& \times\left\langle[O(t), E(\tau)]_{+} V_{+}^{\dagger}(\tau) H_{\text {int },-}\left(\tau_{N}\right) \ldots H_{\text {int },-}\left(\tau_{1}\right)\right\rangle_{0},
\end{aligned}
$$

where we remind the reader that the expectation value $\langle\ldots\rangle_{0}$ is taken with respect to the initial state (30). Eq. (40) contains an $N+1$-point time-ordered correlation function of the combined light-matter system. Using the interaction Hamiltonian (26) and the identity (35), we can break up Eq. (40) into convolutions of field and matter correlation functions,

$$
\begin{aligned}
& \left\langle[O(t), E(\tau)]_{+} V_{+}^{\dagger}(\tau) H_{\text {int },-}\left(\tau_{N}\right) \ldots H_{\text {int },-}\left(\tau_{1}\right)\right\rangle_{0} \\
= & \sum_{v_{1}, \ldots \nu_{N}=0}^{1}\left\langle V_{+}^{\dagger}(\tau) V_{ \pm}^{v_{1}}\left(\tau_{N}\right) \ldots V_{ \pm}^{v_{1}}\left(\tau_{1}\right)\right\rangle_{0}\left\langle[O(t), E(\tau)]_{+} E_{\mp}^{\overline{\nu_{1}}}\left(\tau_{N}\right) \ldots E_{\mp}^{\overline{\nu_{1}}}\left(\tau_{1}\right)\right\rangle_{0} .
\end{aligned}
$$

These field and matter correlation functions can be evaluated separately with the usual methods of quantum chemistry or quantum optics. Here, the summations over $v_{1}, \ldots v_{N}$ indicate the positive or negative frequency component of the operators, respectively, i.e. $V^{0} \equiv V$ and $V^{1} \equiv V^{\dagger}$, and the indices $\overline{v_{1}}, \ldots \overline{v_{N}}$ denote the corresponding opposite components, i.e. $V^{\overline{0}} \equiv V^{\dagger}$ and $V^{\overline{1}} \equiv V$. Note that each commutator of the dipole operator $V_{-}$is accompanied by an anti-commutator of the corresponding field operator, $E_{+}^{\dagger}$, and vice versa. For a more detailed discussion of the properties of these correlation functions, see [89]. The terms with $E_{-}$result in commutators of the light field, signifying the exchange of virtual photons between dipoles [90, 143]. In the linear response, these are related to the optical signal (i.e. the term with $E_{+}$) by the fluctuation-dissipation theorem, yet there is no general relation for nonlinear 
fluctuations [97]. Their contributions are independent of real photons, and in this tutorial, we shall be concerned with quantum correlations and fluctuations that rely solely on the initial state of the fields. We thus only retain the corresponding terms in the following,

$$
\sum_{v_{1}, \ldots \nu_{N}=0}^{1}\left\langle V_{+}^{\dagger}(\tau) V_{-}^{\nu_{1}}\left(\tau_{N}\right) \ldots V_{-}^{\nu_{1}}\left(\tau_{1}\right)\right\rangle_{0}\left\langle[O(t), E(\tau)]_{+} E_{+}^{\overline{\nu_{1}}}\left(\tau_{N}\right) \ldots E_{+}^{\overline{\nu_{1}}}\left(\tau_{1}\right)\right\rangle_{0} .
$$

Eq. (42) was derived in the interaction picture representation, and the time-dependent operator expectation values can be calculated by propagating the coupled quasiparticle equations of motion [92]. In many situations, it is however more convenient to calculate Green's functions in the Schrödinger picture. Defining the time delays $t_{n}=\tau_{n+1}-\tau_{n}$, we can rewrite the sample correlation function as

$$
\left\langle V_{+}^{\dagger}(\tau) V_{-}^{\nu_{1}}\left(\tau_{N}\right) \ldots V_{-}^{\nu_{1}}\left(\tau_{1}\right)\right\rangle_{0}=\left\langle V_{+}^{\dagger}(\tau) \mathcal{G}\left(t_{n}\right) \ldots \mathcal{G}\left(t_{1}\right) V_{-}^{\nu_{1}}\right\rangle_{0},
$$

with the Schrödinger picture propagator

$$
\mathcal{G}(t)=\exp \left[-\frac{i}{\hbar} H_{0-} t\right] .
$$

If the system is coupled to a Markovian bath, we replace the system Hamiltonian in Eq. (44) by the generator of a suitable master equation [61]. Here, we have assumed that the propagator of the ground state prior to the first excitation by light, only produces an irrelevant phase factor. After diagonalization of the system Hamiltonian $H_{0}$, the correlation function can be evaluated in a sum-over-state expansion for sufficiently small systems [94].

Altogether, this perturbation expansion yields $4^{N}$ terms, which quickly become intractable. Yet, due to selection rules and energy conservation, typically only very few of these actually contribute notably to the signal. For instance, in the fourth-order terms, which include measurements of the third-order susceptibiliy $\chi^{(3)}$ and which we will deal with in section 5 , this amounts to $4^{4}=256$ terms already. But in a typical level scheme in molecular aggregates, only adjacent manifolds are dipole coupled [see also Fig. 7], such that at most four terms remain in the end. Clearly, it is very useful to first determine these terms before jumping into heavy numerics. This is done most conveniently by using their diagrammatic representation. A very basic introduction into diagramatics, which focusses on two-dimensional spectroscopy, can be found in [81].

We introduce the diagrams at the hand of the derivation of the linear absorption signal, which is simple enough, such that we can derive it by hand first, and then compare it to the diagrammatic approach. The linear absorption signal is obtained from the expansion of Eq. (38). Exploiting the cyclicity of the trace, $\operatorname{tr}\{V \varrho\}=\operatorname{tr}\{\varrho V\}$, and expanding Eq. (38) to first order in the interaction Hamiltonian (26), we obtain

$$
\begin{array}{r}
S_{\text {int }}^{(1)}(\Gamma)=-\frac{2}{\hbar} \mathfrak{J} \frac{i}{\hbar} \int_{-\infty}^{\infty} d t \int_{t_{0}}^{t} d \tau \operatorname{tr}\left\{E^{\dagger}(t) V(t)\left(E(\tau) V^{\dagger}(\tau)+E^{\dagger}(\tau) V(\tau)\right) \varrho\left(t_{0}\right)\right\} \\
-\operatorname{tr}\left\{E^{\dagger}(t) V(t) \varrho\left(t_{0}\right)\left(E(\tau) V^{\dagger}(\tau)+E^{\dagger}(\tau) V(\tau)\right)\right\},
\end{array}
$$

where the two lines correspond to the two commutator terms. We can see that in the second line a de-excitation operator $V(\tau)$ acts on the initial density matrix $\varrho\left(t_{0}\right)$, thus yielding zero 


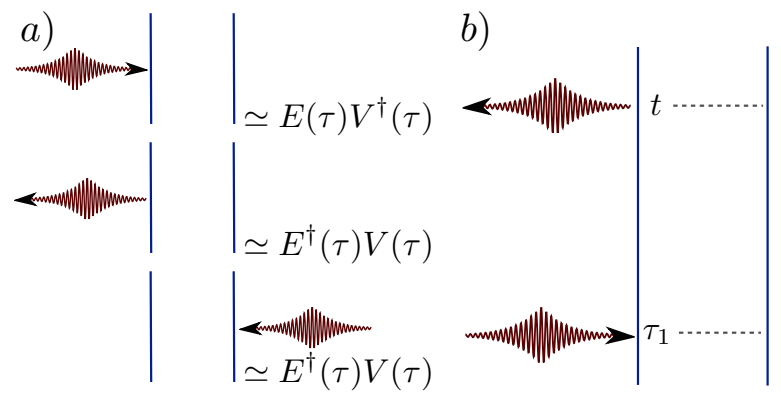

Figure 2. a) The building blocks of the diagrammatic representation of signals: The left and right vertical lines correspond to the evolution of the ket and the bra side of the density matrix, respectively. Each arrow pointing towards (away from) them accounts for an excitation (de-excitation) of the sample, and a de-excitation (excitation) of the field. b) The diagram representing the linear absorption signal.

[compare Eq. (30)]. Likewise, the second term of the first line vanishes, and we arrive at

$$
S_{\text {int }}^{(1)}(\Gamma)=\mathfrak{R} \frac{1}{\hbar^{2}} \int_{-\infty}^{\infty} d t \int_{t_{0}}^{t} d \tau\left\langle V(t) V^{\dagger}(\tau)\right\rangle_{0}\left\langle E^{\dagger}(t) E(\tau)\right\rangle_{0} .
$$

Eq. (46) is the linear absorption signal in the RWA: It connects the two-point correlation function of the sample, $\left\langle V(t) V^{\dagger}(\tau)\right\rangle_{0}$, with the corresponding correlation function of the field, $\left\langle E^{\dagger}(t) E(\tau)\right\rangle_{0}$. We can already learn a very important lesson from this rather straightforward derivation. It shows that any spectroscopic measurement of an $n$-point response function necessarily involves its convolution with the probing field's correlation function of the same order [133]. Hence, quantum correlations cannot have any impact on the the linear absorption signal, since these can only show up in photon correlation measurements - i.e. in fourth-order correlation functions. These are associated with the next higher order signal, where they are connected with the third-order susceptibility $\chi^{(3)}[52,53]$. Thus, they can have an impact on pump-probe or photon echo signals, which measure the same material's $\chi^{(3)}$-susceptibility, provided all the interactions are with the same quantum field.

Having derived this result by hand, we now turn to its diagrammatic representation. The basic building blocks for the diagrammatic representation of optical signals are depicted in Fig. 2a). The time runs from bottom to top. The density matrix $\rho(t)$ is represented by two blue line, which trace the evolution of the ket or the bra side of the matrix, respectively. Each interaction with the light field is depicted by a red arrow. An arrow pointing towards the density matrix describes an excitation of the sample, as well as the corresponding deexcitation of the light field, and vice versa. Thus, as exemplified in the figure, an arrow pointing left corresponds to the application of the operator $E^{\dagger} V$ from the left, if it is a matter de-excitation on the ket-side, and of $E^{\dagger} V$ from the right, if it is a matter excitation on the bra-side. The linear absorption signal (46) is then represented by the diagram in Fig. 2b): The observable's measurement at time $t$ is represented by an outgoing arrow, corresponding to a matter de-excitation $V(\tau)$ and a field excitation $E^{\dagger}(\tau)$. It has to be preceded by the excitation at $\tau$ beforehands. The terms in Eq. (45) which we found to be zero would correspond to a 


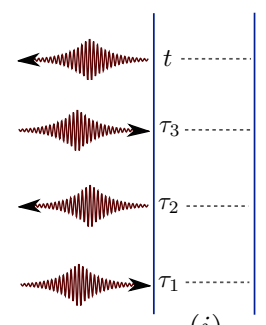

(i)

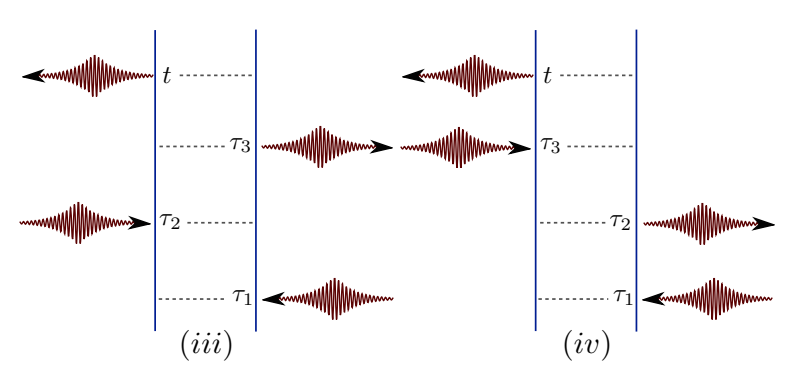

Figure 3. Third-order diagrams contributing to the electromagnetic response of a two-level atom.

diagram, in which the first interaction acts on the right side (the second line), or is given by a de-excitation. In each case, a de-excitation of the ground state indicates immediately that such a process can be neglected. Thus, just drawing the diagram can give us the same information as the explicit calculation carried out above.

In centrosymmetric materials, the second-order contribution vanishes [10], such that the next-leading order in most materials comprises three interactions with the electromagnetic fields. As an example thereof, we depict the third-order diagrams of a two-level atom (or more precisely, of a system consisting of two electronic manifolds at frequencies $\omega_{g}$ and $\omega_{e}$ ) in Fig. $3 .^{+}$We encourage the reader to add the additional diagrams one needs to take into account for a three-manifold system [i.e. including the $f$-states in Eq. (21)].

\section{Photon statistics}

In this section, we shall be concerned with situations in which only a single mode of the light field strongly interacts with the system, such as in a single-mode cavity, or where the description in terms of a single mode is sufficient.

\subsection{Photon counting}

An ideal photodetector is conventionally modelled as a two-level atom, which upon absorption of a photon from the light field is excited into its high-energy state, thus triggering the signal [114]. More advanced theories take into account the finite response time and frequency windows of detectors $[85,86]$, but they do not fundamentally alter the measured quantities.

+ A pedagogical introduction at the hand of this system can also be found in [150]. 

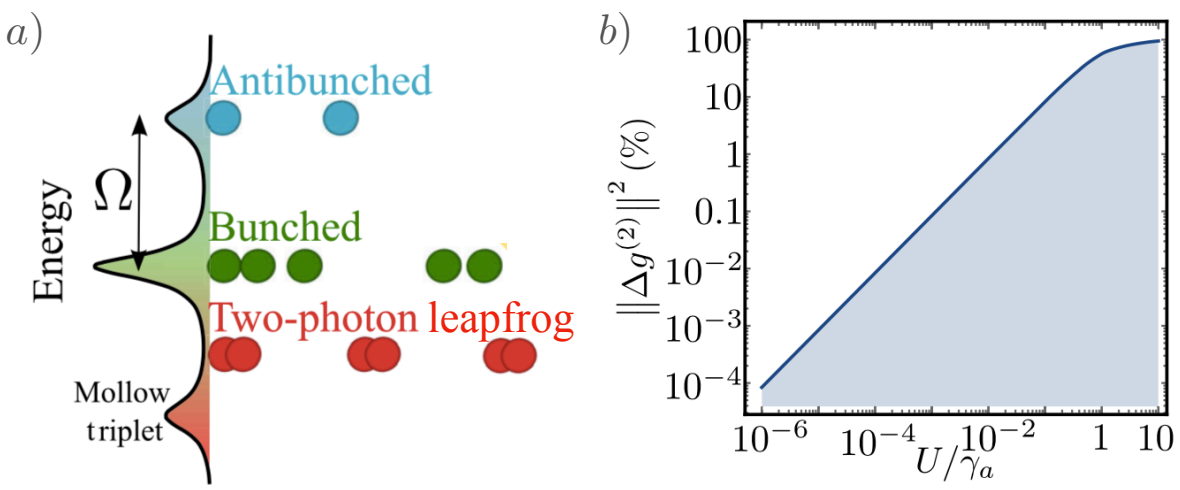

Figure 4. a) The Mollow triplet of a strongly driven two-level atom features different quantum light statistics in its three resonances, bunched, antibunched, and strongly bunched so-called "two-photon leapfrog". b) The deviation of the $g^{(2)}$-signal from the one with vanishing polariton interaction $(U=0)$ is a sensitive measure for the strength of the interaction. Figures are reproduced with permission from [104].

So we refer for further details to [10]. The coincident excitation of $N$ detectors is represented by normally ordered correlation functions of the field,

$$
G^{(N)}\left(\tau_{1}, \ldots, \tau_{N}\right)=\left\langle E^{\dagger}\left(\tau_{1}\right) \ldots E^{\dagger}\left(\tau_{N}\right) E\left(\tau_{N}\right) \ldots E\left(\tau_{1}\right)\right\rangle .
$$

The two-time coincidence measurement is of particular interest, since it is the lowest order to observe correlations between absorption events, and hence test nonclassical features of the light field. One can show that for classical light

$$
G_{c l}^{(2)}\left(\tau_{1}, \tau_{1}\right) \geq G_{c l}^{(2)}\left(\tau_{1}, \tau_{1}+\tau\right)
$$

for any $\tau$. Any quantum state violating the inequality (48) is consequently nonclassical. A Fock state is perhaps the simplest example to violate the inequality. It exhibits photonic antibunching - the detection of a photon in detector 1 renders the probability to detect another one in detector 2 less likely. Conversely, the detection of a photon from a thermal state (whose photon numbers are described by by a Bose-Einstein distribution) makes it more likely to detect a second one - the state shows photon bunching, and is thus classical. Coherent states show no correlations between successive absorption events, since the absorption of a photon does not alter the state.

\subsection{Light fluctuations in spectroscopy}

We have seen in the discussion of Eq. (46) that any $N$-th order correlation function of the sample system is connected to corresponding $N$-order correlation function of the light. Conversely, the detection of a correlation function of the light yields information on the nonlinear optical response of the light emitting system. Hence, the analysis of correlation functions produced by fluorescence light can provide insight into dynamics in or properties of the emitting system, and thus constitute a quantum optical spectroscopic tool. For instance, it could be employed for the characterization of the light-matter coupling in microcavities 
$[99,102]$, or in the strong driving regime [103, 104, 105, 106]. Many applications focus on the elucidation of nonlinear optical properties in the samples [100, 101], which can be interrogated by correlations in multi-photon emission events. As an example thereof, we present recent results [104] on the detection of nonlinearities in polaritonic systems in Fig. 4. The polaritonic Hamiltonian is given by a single-mode version of Eq. (19), where $U$ then denotes the interaction between polaritons. The polaritons are excited by the light emitted from a strongly driven two-level atom. As shown in Fig. 4a), the driving driving splits the atomic resonance into a triplet of transition between dressed states [107], each of which exhibiting distinct photon statistics. Fig. 4b) then demonstrates how $g^{(2)}$-signal of the polariton emission [i.e. the $G^{(2)}$-signal from Eq. (47) normalised by the squared mean photon number] can detect polariton interactions with great sensitivity.

Since the above applications depend on photon correlation measurements, one is tempted to believe that they must constitute truly quantum signals, which cannot be obtained from classical spectroscopic signals (i.e. from laser spectroscopy). However, Koch and co-workers $[108,109]$ showed that this is not quite true: Correlation functions of the form (47) can be rewritten as phase space integrals over the photonic state's Glauber-Sudarshan distribution (we neglect the detection times for now) [110],

$$
G^{(N)}=\int d^{2} \beta P(\beta) \beta^{* N} \beta^{N},
$$

where $\beta$ denotes the complex amplitude of the coherent state representation. Since any optical signal is related to higher-order correlation functions (see the previous section 3.3), it follows that the signal $S_{|\psi\rangle}$ created by any single-mode quantum state $|\psi\rangle$ can be represented by the convolution of signals created by coherent states with amplitude $|\beta\rangle$,

$$
S_{|\psi\rangle}=\int d^{2} \beta P(\beta) S_{|\beta\rangle} .
$$

The mapping (50) can be exploited to reveal features which would otherwise be hidden by fluctuations [111], such as biexciton coherences [112], or weakly bound quasiparticles consisting of three or more electrons and holes [113]. An example of the latter is reproduced in Fig. 5. Fig. 5a) shows the Wigner function (see, e.g. [110]) of a "slanted Schrödinger cat state" - a Schrödinger cat state of two coherent states with distinct amplitudes, which shows nonclassical interference fringes next to the dominant peak. After the mapping (50) of laser signals onto the signal of this squeezed state in panel $b$ ), distinct resonances pertaining from certain quasiparticles can be easily distinguished. These were previously hidden underneath the laser fluctuations.

Thus, if one is able to collect laser signals with controlled amplitude $\beta$, Eq. (50) suggests that any quantum signal can be obtained by appropriate data manipulation. As we shall see in the next section 5, this situation changes when dealing with multimode fields.

\section{Quantum correlations}

Having discussed the role of number fluctuations in the previous section, we now turn to the impact of quantum correlations in multimode fields. We start the section with a quick 

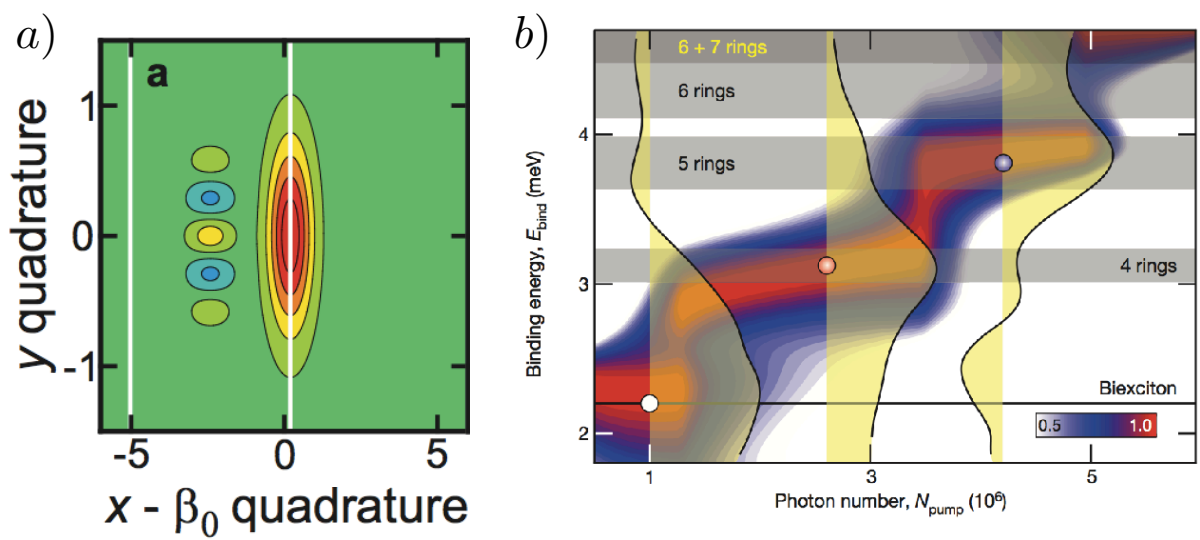

Figure 5. a) Wigner function of a "slanted Schrödinger cat state", with interference fringes visible left of the dominant peak. b) The quantum signal $S_{|\psi\rangle}$ is constructed by convoluting response of laser pulses with the state's Glauber-Sudarshan distribution according to Eq. (50), thus revealing pronounced resonances of weakly bound multi-exciton states that were previously hidden by noise. Figures are reproduced with permission from [113].
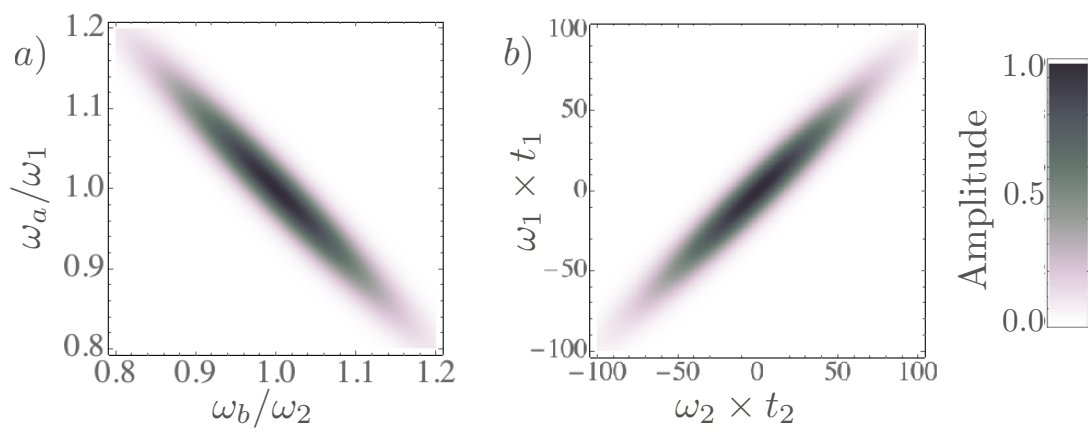

Figure 6. a) The absolute value of the two-photon wavefunction (52) is plotted vs. the two photons' frequencies in case of an anti-correlated correlated state. b) The corresponding time domain signal of the two-photon wavefunction shows positive time correlations. Only an entangled state shows strong correlations in both time and frequency domain.

As parameters, we used $\omega_{1}=\omega_{2}, T_{1}=7 / \omega_{1}, T_{2}=30 / \omega_{1}$, and $\sigma_{p}=0.025 \omega_{1}$. It violates the classical Fourier uncertainty (57), we obtain $\Delta\left(\omega_{a}+\omega_{b}\right) \times \Delta\left(t_{1}-t_{2}\right) \simeq 0.5$.

review of the concept of "time-energy entanglement", as far as it is relevant for spectroscopic applications. A reader who is already familiar with time-energy entanglement could skip this first section.

\subsection{Time-energy entanglement}

A general multimode two-photon state can be written as [84, 115]

$$
|\psi\rangle=\int d \omega_{a} \int d \omega_{b} \Phi\left(\omega_{a}, \omega_{b}\right) a_{1}^{\dagger}\left(\omega_{a}\right) a_{2}^{\dagger}\left(\omega_{b}\right)|0\rangle
$$

with the two-photon amplitude $\Phi\left(\omega_{a}, \omega_{b}\right)$. Note that in general this state cannot be described by a single mode as in the previous section. We have introduced indexed field operators 
to indicate that we assume that the two photons can be distinguished by their propagation direction or their polarization. In particular, we shall focus on two-photon states created by type-II downconversion, where we can write the two-photon amplitude as

$$
\Phi\left(\omega_{a}, \omega_{b}\right)=\alpha \exp \left[\left(\omega_{a}+\omega_{b}-\omega_{p}\right)^{2} /\left(2 \sigma_{p}^{2}\right)\right] \exp \left[-\left(\Delta k\left(\omega_{a}, \omega_{b}\right) L\right)^{2}\right] .
$$

Since it cannot be described by a single mode, this state is sometimes referred to as a two-mode squeezed state (the two modes pertaining to the two orthogonal polarization vectors of the photons in type-II downconversion). The form of this two-photon amplitude can be understood as a consequence of the two-photon creation process in spontaneous downconversion. A pump laser is directed onto birefringent material, where a pump photon can split spontaneously into two photons. This process is subject to energy and momentum conservation, which each account for one of the two exponentials in Eq. (52). The first exponential in Eq. (52) reflects energy conservation: The sum of the two photon frequencies has to add up to the frequency of the destroyed pump photon. The finite pump bandwidth $\sigma_{p}$ thus determines the distribution of this sum, and can thus create frequency anti-correlations between the two photons. If the first photon has the frequency $\omega_{p} / 2+x$, the second photon must have the frequency $\omega_{p} / 2-x$. The second exponential reflects momentum conservation, which limits the possible phase matching frequencies. The downconversion process can take place at any point along the length $L$ of the pump's transition during the crystal. Depending on the frequencies $\omega_{a}$ and $\omega_{b}$, the downconverted photons travel at different group velocities, thus acquiring a time delay until they exit the crystal. This effect increases with increasing frequency difference between $\omega_{a}$ and $\omega_{b}$, and thus creates positive frequency correlations. We can write approximately [28]

$$
\Delta k\left(\omega_{a}, \omega_{b}\right) L=\gamma\left[\left(\omega_{a}-\omega_{p} / 2\right) T_{1}+\left(\omega_{b}-\omega_{p} / 2\right) T_{2}\right],
$$

with $\gamma=0.22$. The two parameters $T_{1}$ and $T_{2}$ depend on the material (and need not be positive), and here we only consider degenerate downconversion, where both photon frequencies are centred around $\omega_{p} / 2$.

The state (51) is called separable, if the two-photon amplitude factorizes into $\Phi\left(\omega_{a}, \omega_{b}\right)=$ $\phi_{1}\left(\omega_{1}\right) \phi_{2}\left(\omega_{2}\right)$. Otherwise, it is entangled. The standard approach to analyze the entanglement properties of this state involves its singular value decomposition $[116,117,118]$, which reads

$$
\Phi\left(\omega_{a}, \omega_{b}\right)=\sum_{k} r_{k} \psi_{k}\left(\omega_{a}\right) \phi_{k}\left(\omega_{b}\right) .
$$

The nonzero coefficients $r_{k}$ - called Schmidt coefficients - yield the effective number of dimensions available for quantum information purposes [116]. The functions $\psi_{k}$ and $\phi_{k}$ are the corresponding Schmidt modes. In the case of the two-photon amplitude (52), the singular value decomposition can be carried out analytically [119], and the Schmidt modes are simply given by Hermite functions. This allows for a very speedy calculation of the field correlation functions, even in a high-intensity regime. Details can be found in [93], and are not repeated here. The application to quantum information of such broadband quantum light has been intensely discussed in [120].

Unfortunately, their application in quantum information processing does not necessarily tell us much about the possible use of time-frequency entangled photons in spectroscopy, 
where different properties may be important. For instance, if one's goal consists in creating a pure Bell state in the polarization degrees of freedom, time-energy entanglement can in fact be detrimental, and should be minimised [117]. We therefore turn back to Eqs. (51) and (52). Depending on the parameters of the pump pulse and the birefringent material, one of the two exponentials in Eq. (52) dominates the photonic wavefunction - resulting in, respectively, frequency correlated or anti-correlated photon pairs. An example of the latter case is shown in Fig. 6a) as a function of the two frequencies $\omega_{a}$ and $\omega_{b}$. Each are measured in units of their centre frequency $\omega_{1}$ and $\omega_{2}$. Measuring the frequency of one photon, say $\omega_{a}$, will result in a broad distribution, corresponding to the marginal of the distribution along the $\omega_{a}$-axis. But once a value is measured, the wavefunction of the other photon collapses onto a frequency $\omega_{b}$, such that their sum adds up to the pump frequency $\omega_{p}$.

Strong frequency correlations pre se are however not a sign of quantum correlations. Indeed, if we replace the pure quantum state (51) by a density matrix, in which we erased the off-diagonal elements,

$$
\varrho_{c l}=\int d \omega_{a} \int d \omega_{b}\left|\Phi\left(\omega_{a}, \omega_{b}\right)\right|^{2} a_{1}^{\dagger}\left(\omega_{a}\right) a_{2}^{\dagger}\left(\omega_{b}\right)|0\rangle\langle 0| a_{2}\left(\omega_{b}\right) a_{1}\left(\omega_{a}\right),
$$

we still obtain the same frequency correlations shown in Fig. 6a). However, the in contrast to Eq. (51), the density matrix (55) describes a time-independent photon distribution. Thus, in deleting the off-diagonal matrix elements, we have thrown away the time correlations encoded in Eq. (51). The time domain of Eq. (51), given by

$$
\Phi\left(t_{1}, t_{2}\right)=\int d \omega_{a} \int d \omega_{b} \Phi\left(\omega_{a}, \omega_{b}\right) e^{-i\left(\omega_{a} t_{1}+\omega_{b} t_{2}\right)},
$$

is plotted in Fig. 6b). Frequency anti-correlations imply positive correlations in time. The broad distribution long the diagonal corresponds to the long duration of the pump pulse creating the photon pair, and the narrow distribution along the anti-diagonal stem from the pairs arriving simultaneously. It shows that the defining feature of quantum correlations are the simultaneous time and frequency correlations. Any application of quantum light that claims to rely on quantum correlations has to make use of both time and frequency correlations. Otherwise, our reasoning shows that we could obtain the same result with classical correlations. More precisely, we can say that classical light is bounded by the inequality [128]

$$
\Delta\left(\omega_{a}+\omega_{b}\right) \times \Delta\left(t_{1}-t_{2}\right) \geq 1,
$$

where $\Delta\left(\omega_{a}+\omega_{b}\right)$ denotes the uncertainty in the sum frequency, and $\Delta\left(t_{1}-t_{2}\right)$ the uncertainty in the time delay between the arrivals of the two photons. Crudely speaking, this is the Fourier theorem for two-photon transitions: what you gain in frequency resolution is what you lose in time resolution, and vice versa. With entangled photons, however, the frequency uncertainty is given by the pump bandwidth $\sigma_{p}$, while the time uncertainty depends on the arrival times of the photons, $\sim T_{2}-T_{1}$. Both are independent quantities, and hence, entangled photons can violate the above inequality [122]. 


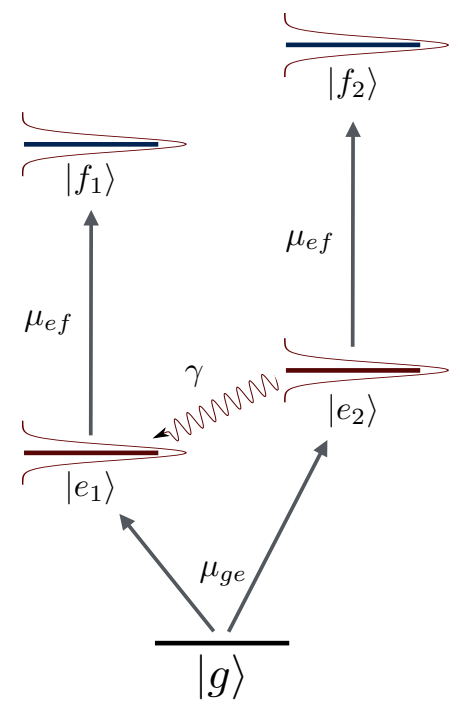

Figure 7. The toy model employed here: A single electronic ground state $|g\rangle$ is dipole-coupled to two single exciton states $\left|e_{1}\right\rangle$ and $\left|e_{2}\right\rangle$. Each of these is in turn coupled to a distinct twoexciton state $\left|f_{1}\right\rangle$ and $\left|f_{2}\right\rangle$, respectively. As indicated, all the excited states are broadened by the coupling to an environment described by the rate $\gamma$, which further causes rapid population transfer from $\left|e_{2}\right\rangle$ to $\left|e_{1}\right\rangle$.

The eigenenergies are set to $\hbar \omega_{e_{1}}=1.4 \mathrm{eV}, \hbar \omega_{e_{2}}=1.55 \mathrm{eV}, \hbar \omega_{f_{1}}=2.7 \mathrm{eV}$, and $\hbar \omega_{f_{2}}=2.9 \mathrm{eV}$. The resonance broadening is $\hbar \gamma=0.05 \mathrm{eV}$, and the population transfer rate $\kappa=1 / 10 \mathrm{fs}$. The dipole moments are all identical, $\mu_{g e}=\mu_{e f}=\mu$.

\subsection{Two-photon induced fluorescence}

We will examine the impact of quantum correlations on two-photon absorption processes at the hand of the toy model in Fig. 7. It consists of a single electronic ground state $|g\rangle$, which is connected to two single exciton states $\left|e_{1}\right\rangle$ and $\left|e_{2}\right\rangle$, that in turn are dipole coupled to double exciton states $\left|f_{1}\right\rangle$ and $\left|f_{2}\right\rangle$. In the last section, we have established that simultaneous time and frequency correlations are the hallmark of time-energy entanglement. Thus, to test the time correlations, we add a decay channel which incoherently transfers population from the higher-energetic state $\left|e_{2}\right\rangle$ to $\left|e_{1}\right\rangle$, and hence renders the dynamics irreversible. We model this transfer with a Pauli rate equation,

$$
\begin{aligned}
& \frac{d}{d t} p_{e_{1}}=\kappa\left(-a p_{e_{1}}+p_{e_{2}}\right) \\
& \frac{d}{d t} p_{e_{2}}=\kappa\left(a p_{e_{1}}-p_{e_{2}}\right),
\end{aligned}
$$

with $a=1 / 20$ the equilibrium population of $p_{e_{2}}$, and $\kappa=1 / 10 f s^{-1}$. To efficiently excite the state $\left|f_{2}\right\rangle$, one needs to suppress this decay channel. At the same time, one would like to minimise the bandwidth of the involved pulses to avoid the accidental excitation of $\left|f_{1}\right\rangle$. This is best accomplished by strong time and frequency correlations.

The simplest optical signal to investigate population distributions are the fluorescence signals we derived in section 3.2. Since electronic coherences typically decay much faster than electronic populations, the fluorescence is created by electronic populations. Eq. (39) for 


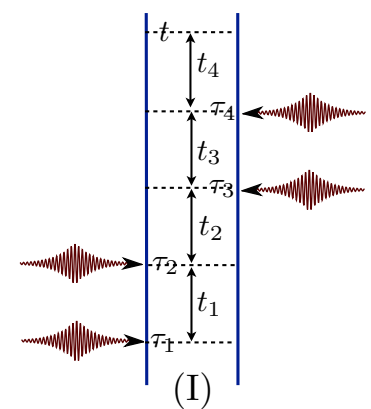

(I)

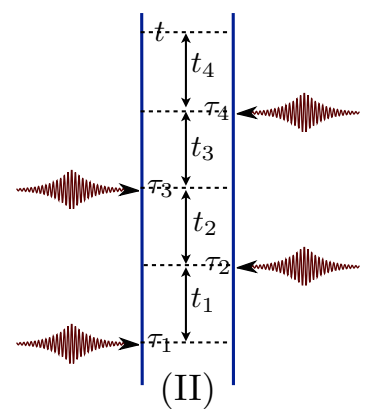

(II)



(III)

Figure 8. Diagrams yielding the population in the $f$-manifold. Their complex conjugates are obtained by reflecting the arrows along the horizontal axis.

the fluorescence from the $f$-populations - the two-photon induced fluorescence (TPIF) signal - then simplifies to [94],

$$
S_{T P I F}(\omega ; \Gamma)=\sum_{e, f}\left|\mu_{e f}\right|^{2} \delta\left(\omega-\omega_{f}+\omega_{e}\right) \int d t p_{f}(t ; \Gamma),
$$

where the summations run over all doubly excited states $|f\rangle$ and all single excited states $|e\rangle$. The signal is detected at the frequency $\omega$. After integrating over this detection frequency, we obtain the two-photon fluorescence action spectrum,

$$
S_{a c t}(\Gamma)=\sum_{f}\left|\mu_{e f}\right|^{2} \int d t p_{f}(t ; \Gamma)
$$

Thus, we need to calculate the time-integrated population in state $|f\rangle$, with $p_{f}(t ; \Gamma)=$ $\operatorname{tr}\left\{P_{f} \varrho(t)\right\}$ the expectation value of the projection operator $P_{f}=|f\rangle\langle f|$. This population at time $t$ is given by the three diagrams in Fig. 8, as well as their complex conjugates,

$$
p_{f}(t ; \Gamma)=\mathfrak{R}\left\{p_{f,(I)}(t ; \Gamma)+p_{f,(I I)}(t ; \Gamma)+p_{f,(I I I)}(t ; \Gamma)\right\},
$$

where the three parts read, respectively,

$$
\begin{aligned}
& p_{f,(I)}(t ; \Gamma)=\left(-\frac{i}{\hbar}\right)^{4} \int_{0}^{\infty} d t_{4} \int_{0}^{\infty} d t_{3} \int_{0}^{\infty} d t_{2} \int_{0}^{\infty} d t_{1} \\
& \times \sum_{e, e^{\prime}} \mathcal{G}_{f f}\left(t_{4}\right) \mu_{e^{\prime} f} \mathcal{G}_{f e^{\prime}}\left(t_{3}\right) \mu_{g e^{\prime}} \mathcal{G}_{f g}\left(t_{2}\right) \mu_{e f} \mathcal{G}_{e g}\left(t_{1}\right) \mu_{g e} \\
& \times\left\langle E^{\dagger}\left(t-t_{4}-t_{3}\right) E^{\dagger}\left(t-t_{4}\right) E\left(t-t_{4}-t_{3}-t_{2}\right) E\left(t-t_{4}-t_{3}-t_{2}-t_{6}(9) .\right)\right. \\
& p_{f,(I I)}(t ; \Gamma)=\left(-\frac{i}{\hbar}\right)^{4} \int_{0}^{\infty} d t_{4} \int_{0}^{\infty} d t_{3} \int_{0}^{\infty} d t_{2} \int_{0}^{\infty} d t_{1} \\
& \times \sum_{e, e^{\prime}, e^{\prime \prime}} \mathcal{G}_{f f}\left(t_{4}\right) \mu_{e^{\prime \prime} f} \mathcal{G}_{f e^{\prime \prime}}\left(t_{3}\right) \mu_{e^{\prime \prime} f} \mathcal{G}_{e e^{\prime} ; e^{\prime \prime} e^{\prime \prime}}\left(t_{2}\right) \mu_{g e^{\prime}} \mathcal{G}_{e g}\left(t_{1}\right) \mu_{g e} \\
& \times\left\langle E^{\dagger}\left(t-t_{4}-t_{3}\right) E^{\dagger}\left(t-t_{4}\right) E\left(t-t_{4}-t_{3}-t_{2}\right) E\left(t-t_{4}-t_{3}-t_{2}-t_{6}(6) .\right)\right. \\
& p_{f,(I I)}(t ; \Gamma)=\left(-\frac{i}{\hbar}\right)^{4} \int_{0}^{\infty} d t_{4} \int_{0}^{\infty} d t_{3} \int_{0}^{\infty} d t_{2} \int_{0}^{\infty} d t_{1} \\
& \times \sum_{e, e^{\prime}, e^{\prime \prime}} \mathcal{G}_{f f}\left(t_{4}\right) \mu_{e^{\prime \prime} f} \mathcal{G}_{e^{\prime \prime} f}\left(t_{3}\right) \mu_{e^{\prime \prime} f} \mathcal{G}_{e e^{\prime} ; e^{\prime \prime} e^{\prime \prime}}\left(t_{2}\right) \mu_{g e^{\prime}} \mathcal{G}_{e g}\left(t_{1}\right) \mu_{g e} \\
& \times\left\langle E^{\dagger}\left(t-t_{4}-t_{3}\right) E^{\dagger}\left(t-t_{4}\right) E\left(t-t_{4}-t_{3}-t_{2}\right) E\left(t-t_{4}-t_{3}-t_{2}-t_{0.55}\right)\right.
\end{aligned}
$$


Since we are interested in the time-integrated population in Eq. (60), it is beneficial to evaluate the diagrams in frequency domain, where the final time integration yields a delta-function. We arrive at

$$
\begin{aligned}
\int d t p_{f,(I)}(t ; \Gamma) & =\left(-\frac{i}{\hbar}\right)^{4} \int \frac{d \omega_{a}^{\prime}}{2 \pi} \int \frac{d \omega_{b}^{\prime}}{2 \pi} \int \frac{d \omega_{a}}{2 \pi} \int \frac{d \omega_{b}}{2 \pi} 2 \pi \delta\left(\omega_{a}+\omega_{b}-\omega_{a}^{\prime}-\omega_{b}^{\prime}\right) \\
& \times \sum_{e, e^{\prime}} \mathcal{G}_{f f}\left(\omega_{a}+\omega_{b}-\omega_{a}^{\prime}-\omega_{b}^{\prime}\right) \mu_{e^{\prime} f} \mathcal{G}_{f e^{\prime}}\left(\omega_{a}+\omega_{b}-\omega_{a}^{\prime}\right) \\
& \times \mu_{g e^{\prime}} \mathcal{G}_{f g}\left(\omega_{a}+\omega_{b}\right) \mu_{e f} \mathcal{G}_{e g}\left(\omega_{a}\right) \mu_{g e}\left\langle E^{\dagger}\left(\omega_{a}^{\prime}\right) E^{\dagger}\left(\omega_{b}^{\prime}\right) E\left(\omega_{b}\right) E\left(\omega_{d}\right) 6\right)
\end{aligned}
$$

and analogous expression for the other two diagrams. Using the singular value decomposition (54) of the two-photon state, the remaining three frequency integrals can be evaluated numerically (for details, see [93]).

In Fig. 9, the TPIF action spectrum (61) is shown versus the pump frequency $\omega_{p}$ creating the entangled photon pair (solid line). Two strong resonances indicate resonant two-photon transitions to the respective $f$-state, when the sum of the two photons' frequencies match the $f$-state's frequency, i.e. $\omega_{p}=\omega_{f}$. The corresponding TPIF signal (60) is depicted in Fig. 9b), where the detection frequency $\omega$ forms the second axis. The two resonances seen in the action spectrum fluoresce at slightly different frequencies. The TPIF signal with entangled photons clearly reveals the full two-exciton level structure.

We also depict the TPIF signal created by laser pulses with Gaussian envelopes,

$$
E(\omega)=A_{0} e^{-\left(\omega-\omega_{0}\right)^{2} /\left(2 \sigma^{2}\right)},
$$

as dashed plots in Fig. 9a). To enable a direct comparison with entangled photons, they are depicted versus twice their centre frequencies $2 \times \omega_{0}$. The bandwidth $\sigma$ is fixed, such that it either reproduces the entangled photon bandwidths (corresponding to the marginals in Fig. 6) or the frequency resolution in the $f$-manifold (corresponding to their diagonal). The purple plot corresponds to the former case, the green one to the latter. In both cases, a resonance around $2 \omega_{0}=\omega_{f_{1}}$ dominates the signal. It is broadened more strongly than in the entangled photon case, which stems from the interference between $g-e$ and the $e-f$ transitions. While in the entangled photon case, the $g-e$ transitions are broadened by the large bandwidth of the individual photons, and only the $e-f$ transitions are well defined. This is not possible in the absence of correlations, and the resulting signal is broadened [93].

More importantly, however, laser pulses do not show a clear resonance pertaining to the state $\left|f_{2}\right\rangle$. Since the intermediate state $\left|e_{2}\right\rangle$ is depleted very quickly, it is very hard to efficiently excite the ladder $|g\rangle \rightarrow\left|e_{2}\right\rangle \rightarrow\left|f_{2}\right\rangle$, and the resonance of $\left|f_{2}\right\rangle$ only shows up as a weak shoulder on the dominant $\left|f_{1}\right\rangle$-resonance. Classical frequency correlations cannot help either, since - as we discussed earlier - it is necessary to have both time and frequency correlations. It is very hard to detect the signal from $\left|f_{2}\right\rangle$ using classical spectroscopy, because the state hardly gets excited to begin with. Hence, spreading the signal in two dimensions, as in multidimensional spectroscopy $[1,2,3]$, or any form of data manipulation, as seen in the previous section 4 , will necessarily not be helpful: The two-photon state in Fig. 6 is described by the coherent superposition of approx. seventy Schmidt modes with considerable weights $r_{k}$. Reproducing the optical signal created by this state with classical signals is clearly not feasible in practice 

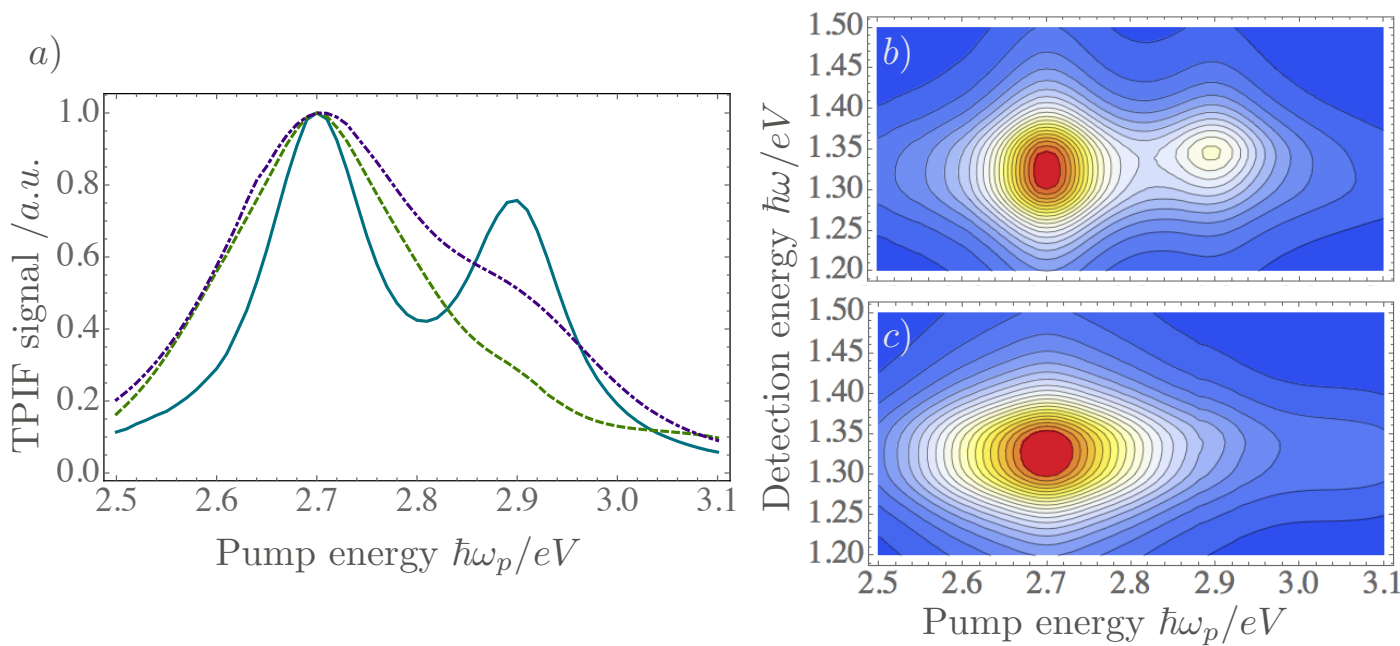

Figure 9. a) The TPIF action spectrum (61) induced by entangled photons (blue, solid), and laser pulses with the roughly same frequency resolution (green, dashed) or the same bandwidth (red, dot-dashed) as the entangled photons with the same parameters as in Fig. 6a). b) Frequency-dispersed TPIF signal (60) of the entangled photon signal shown in a). c) Frequency-dispersed TPIF signal of laser pulses [green, dashed plot in a)].

due to the large number of Schmidt modes with increasingly complex spectral structure, where a multidimensional generalisation of Eq. (50) would require an integration over a $\sim 2 \times 70^{2}$ dimensional phase space. Yet it is also impossible in principle, as one would need to reproduce the signal from coherences between distinct modes.

Of course, the toy model shown here was designed to exhibit the advantages of entangled photon spectroscopy in a very pronounced way. Yet in realistic models of photosynthetic molecular aggregates, the same physics can be employed to control exciton distributions $[121,122]$. The impact of frequency correlations in two-photon transitions was also discussed in the context of quantum dots [123], or in the excitation of vibronic states [124], opening the possibility to control chemical reactions [125]. Internal conversion processes can also be investigated [126].

\subsection{Multidimensional spectroscopic signals}

So far, we have only discussed phase-insensitive signals, i.e. signals that do not depend on the phase of the involved light fields. Given the tremendous success of multidimensional laser spectroscopy we had mentioned in the introduction, it would certainly be very appealing to combine the strong correlation effect with multidimensional techniques. A viable approach to achieve this goal was proposed in [128], where fluorescence-based two-dimensional spectroscopy - well established in laser spectroscopy [129] - is combined with entangled photon absorption. This novel optical signal was used to simulate the spectroscopy of configurations of a molecular dimer [128]. Apart from the simultaneous time and frequency resolution discussed in the previous section, the authors note the "suppression of diagonal 2D spectral features, and the enhancement and narrowing of off-diagonal spectral cross- 
peaks that contain information about electronic couplings", all of which would render twodimensional entangled photon spectroscopy an appealing tool to extract electronic couplings in complex molecular aggregates. Similar approaches were also suggested for absorptionbased measurements [52,54]. It was later pointed out [132] that some of these effects could also be obtained using classical light together with an appropriate combination of time delays. Yet regardless of whether, or to what extent, this signal is truly quantum in nature, the practical usefulness of such a technique is beyond doubt.

\section{Conclusions}

We have provided an introduction into the emerging field of quantum spectroscopy, and especially of entangled photon spectroscopy. After reviewing the background of quantum electrodynamics in section 2 and of the construction of nonlinear optical signals in section 3 , we had looked at two main branches of quantum spectroscopy - photonic fluctuations in a single mode and quantum correlations in a multimode situation. We saw that in the former case, it is possible to project classical signals* onto quantum responses, and thereby reveal weak features in the signal. This was no longer feasible in the multimode regime, where we found the violation of the classical Fourier uncertainty (57) in two-photon transitions by entangled photons to be a possible source for their use to control excited state distributions.

In focussing on specific examples, we were forced to neglect a number of different applications of entangled photon spectroscopy. Besides the possible role of entangled photons in Raman measurements [57, 141], this includes the use of photon coincidence counting as a new measurement tool. In this application, as described at the example of Yabushita and co-workers [43], one photon of the entangled pair interacts with the sample, while the other is detected in coincidence. This is explored in a number of experimental $[145,146,147,149,148]$ and theoretical papers $[86,85,96,10]$. It was however pointed out recently that it is the frequency correlations rather than entanglement per se that is responsible for this application [131]. Hence, one could in principle think of obtaining similar effects with classical, correlated light, and thus classify it as a classical form of spectroscopy.

Furthermore, in this theoretically minded tutorial, we have not discussed the very relevant question of the excitation efficiency at small photon fluxes. Even though, under the right circumstances, quantum correlations effects can prevail even at large average photon numbers $[142,130]$, these effects are typically diminished at large photon fluxes. This efficiency is greatly affected by photon correlations [134, 135], and could be further enhanced in nanoantenna [127], or by frequency filtering [137]. Since the shaping of entangled photon pairs was already demonstrated in 2004 [44], and so far employed for quantum information

\footnotetext{
* It should be pointed out that even the present definition of "classical light" ( large amplitude, coherent states) is subject of a current debate [143, 144]. Furthermore, a nonclassical state does not necessarily produce a nonclassical signal: Highly nonclassical states, such as an $N$-photon Fock state, can produce nonlinear optical signals which - apart from the scaling $\left(\propto\langle\hat{n}\rangle^{2}\right.$ for a coherent state, $\propto N(N-1)$ for the Fock state) - are indistinguishable from "classical" signals [53]. To the best of our knowledge, there is no consensus regarding whether or not one is to label such signals as quantum spectroscopy.
} 
purposes $[138,139,140]$, this opens the possibility of further enhancing the efficiency using coherent control techniques. In fact, it can be rigorously shown that quantum correlations can enhance the efficiency beyond the classically achievable limit [136].

Even though entangled photon sources were developed almost thirty years ago, the systematic investigation of the possible use of entanglement and of quantum fluctuations in optical signals has really gained momentum in recent years only. This is still an emerging field, with open questions ranging from fundamental questions on the unambiguous distinction of quantum properties in optical signals to the very practical problem on how to best exploit the quantum features for a given sample system.

\section{Acknowledgments}

I acknowledge funding from the European Research Council under the European Union's Seventh Framework Programme (FP7/2007-2013) Grant Agreement No. 319286 Q-MAC.

[1] D. M. Jonas, Two-Dimensional Femtosecond Spectroscopy, Ann. Rev. Phys. Chem. 54, 425-463 (2003).

[2] M. Cho, Coherent Two-Dimensional Optical Spectroscopy, Chem. Rev. 108, 1331-1418 (2008).

[3] S. Mukamel, D. Abramavicius, L. Yang, W. Zhuang, I. Schweigert and D. V. Voronine, Coherent Multidimensional Optical Probes for Electron Correlations and Exciton Dynamics: From NMR to Xrays, Acc. Chem. Res. 42, 553-562 (2009).

[4] A. Ishizaki and G. R. Fleming, Quantum Coherence in Photosynthetic Light Harvesting, Ann. Rev. Cond. Mat. 3, 333-361 (2012).

[5] A. Chenu and G. D. Scholes, Coherence in Energy Transfer and Photosynthesis, Ann. Rev. Phys. Chem. 66, 69-96 (2015).

[6] G. D. Scholes et al., Using coherence to enhance function in chemical and biophysical systems, Nature $\mathbf{5 4 3}, 647$ ?656 (2017).

[7] Y. Silberberg, Quantum Coherent Control for Nonlinear Spectroscopy and Microscopy, Ann. Rev. Phys. Chem. 60, 277-292 (2009).

[8] S. Hoyer et al., Realistic and verifiable coherent control of excitonic states in a light-harvesting complex, New J. Phys. 16, 045007 (2014).

[9] I. A. Walmsley, Quantum optics: Science and technology in a new light, Science 348, 525-530 (2015).

[10] K. E. Dorfman, F. Schlawin and S. Mukamel, Nonlinear optical signals and spectroscopy with quantum light, Rev. Mod. Phys. 88, 045008 (2016).

[11] G. S. Engel et al., Evidence for wavelike energy transfer through quantum coherence in photosynthetic systems, Nature 446, 782 - 786 (2007).

[12] S. M. Falke et al., Coherent ultrafast charge transfer in an organic photovoltaic blend, Science 344, 10011005 (2013).

[13] I. Kassal, J. Yuen-Zhou and S. Rahimi-Keshari, Does Coherence Enhance Transport in Photosynthesis?, J. Phys. Chem. Lett. 4, 362-367 (2013).

[14] P. Brumer and M. Shapiro, Molecular Response in One-Photon Absorption via Natural Thermal Light vs. Pulsed Laser Excitation, Proc. Natl. Acad. Sci. USA, 109, 19575 (2012).

[15] T. V. Tscherbul and P. Brumer, Long-Lived Quasistationary Coherences in a V-type System Driven by Incoherent Light, Phys. Rev. Lett. 113, 113601 (2014).

[16] R. J. Glauber, Quantum Theory of Optical Coherence (Wiley, Hoboken, USA 2007)

[17] J. Olsina, A. G. Dijkstra, C. Wang and J. Cao, Can Natural Sunlight Induce Coherent Exciton Dynamics?, arXiv: 1408.5385

[18] A. Chenu, A. M. Branczyk and J. E. Sipe, First-order decomposition of thermal light in terms of a statistical mixture of single pulses, Phys. Rev. A 91, 063813 (2015). 
[19] A. Chenu and P. Brumer, Transform-limited-pulse representation of excitation with natural incoherent light, J. Chem. Phys. 144, 1063 (2016).

[20] R. J. Glauber, The Quantum Theory of Optical Coherence, Phys. Rev. 130, 2529-2539 (1963).

[21] B. R. Mollow, Two-Photon Absorption and Field Correlation Functions, Phys. Rev. 175, 1555-1563 (1968).

[22] J. Gea-Banacloche, Two-photon absorption of nonclassical light, Phys. Rev. Lett. 62, 1603-1606 (1989).

[23] J. Javanainen and P. L. Gould, Linear intensity dependence of a two-photon transition rate, Phys. Rev. A 41, 5088-5091 (1990).

[24] B. E. A. Saleh, B. M. Jost, H.-B. Fei and M. C. Teich, Entangled-Photon Virtual-State Spectroscopy, Phys. Rev. Lett. 80, 3483-3486 (1998).

[25] E. S. Polzik, J. Carri and H. J. Kimble, Spectroscopy with squeezed light, Phys. Rev. Lett. 68, 3020-3023 (1992).

[26] N. Ph. Georgiades, E. S. Polzik, K. Edamatsu, H. J. Kimble, and A. S. Parkins, Nonclassical Excitation for Atoms in a Squeezed Vacuum, Phys. Rev. Lett. 75, 3426-3429 (1995).

[27] N. Ph. Georgiades, E. S. Polzik and H. J. Kimble, Atoms as nonlinear mixers for detection of quantum correlations at ultrahigh frequencies, Phys. Rev. A 55, R1605-R1608 (1997).

[28] Y. Shih, Entangled biphoton source - property and preparation, Rep. Prog. Phys. 66, 1009 (2003).

[29] B. Dayan, Theory of two-photon interactions with broadband down-converted light and entangled photons, Phys. Rev. A 76, 043813 (2007).

[30] I. Ali Khan and J. C. Howell, Experimental demonstration of high two-photon time-energy entanglement, Phys. Rev. A 73, 031801 (2006).

[31] J.-W. Pan, Z.-B. Chen, C.Y. Lu, H. Weinfurter, A. Zeilinger and M. Zukowski, Multiphoton entanglement and interferometry, Rev. Mod. Phys. 84, 777-838 (2012).

[32] T. B. Pittman, Y. H. Shih D. V. Strekalov and A. V. Sergienko, Optical imaging by means of two-photon quantum entanglement, Phys. Rev. A 52, R3429-R3432 (1995).

[33] R. S. Bennink, S. J. Bentley and R. W. Boyd, "Two-Photon” Coincidence Imaging with a Classical Source, Phys. Rev. Lett. 89, 113601 (2002).

[34] R. Kaltenbaek, J. Lavoie, D. N. Biggerstaff and K. J. Resch, Quantum-inspired interferometry with chirped laser pulses, Nature Phys. 4, 864-868 (2008).

[35] R. Kaltenbaek, J. Lavoie and K. J. Resch, Classical Analogues of Two-Photon Quantum Interference, Phys. Rev. Lett. 102, 243601 (2009).

[36] J. D. Franson, Nonlocal cancellation of dispersion, Phys. Rev. A 45, 3126-3132 (1992).

[37] A. M. Steinberg, P. G. Kwiat and R. Y. Chiao, Dispersion cancellation and high-resolution time measurements in a fourth-order optical interferometer, Phys. Rev. A 45, 6659-6665 (1992).

[38] T. S. Larchuk, M. C. Teich and B. E. A. Saleh, Nonlocal cancellation of dispersive broadening in MachZehnder interferometers, Phys. Rev. A 52, 4145-4154 (1995).

[39] A. F. Abouraddy et al. Quantum-optical coherence tomography with dispersion cancellation, Phys. Rev. A 65, 053817 (2002).

[40] M. B. Nasr, B. E. A. Saleh, A. V. Sergienko and M. C. Teich, Demonstration of Dispersion-Canceled Quantum-Optical Coherence Tomography, Phys. Rev. Lett. 91, 083601 (2003).

[41] A. N. Boto et al., Quantum Interferometric Optical Lithography: Exploiting Entanglement to Beat the Diffraction Limit, Phys. Rev. Lett. 85, 2733-2736 (2000).

[42] M. D'Angelo, M. V. Chekhova and Y. Shih, Two-Photon Diffraction and Quantum Lithography, Phys. Rev. Lett. 87, 013602 (2001).

[43] A. Yabushita and T. Kobayashi, Spectroscopy by frequency-entangled photon pairs, Phys. Rev. A 69, 013806 (2004).

[44] B. Dayan, A. Pe'er, A. A. Friesem and Y. Silberberg, Two Photon Absorption and Coherent Control with Broadband Down-Converted Light, Phys. Rev. Lett. 93, 023005 (2004).

[45] B. Dayan, A. Pe'er, A. A. Friesem and Y. Silberberg, Nonlinear Interactions with an Ultrahigh Flux of Broadband Entangled Photons, Phys. Rev. Lett. 94, 043602 (2005).

[46] B. Dayan, A. Pe'er, A. A. Friesem and Y. Silberberg, Temporal Shaping of Entangled Photons, Phys. Rev. 
Lett. 94, 073601 (2005).

[47] R. de J. Leon-Montiel, J. Svozilok, L. J. Salazar-Serrano and J. P. Torres, Role of the spectral shape of quantum correlations in two-photon virtual-state spectroscopy, New J. Phys. 15, 053023 (2013).

[48] D.-I. Lee and T. Goodson, Entangled Photon Absorption in an Organic Porphyrin Dendrimer, J. Phys. Chem. B 110, 25582 (2006).

[49] L. Upton et al., Optically Excited Entangled States in Organic Molecules Illuminate the Dark, J. Phys. Chem. Lett. 12, 2046-2052 (2013).

[50] A. R. Guzman et al., Spatial Control of Entangled Two-Photon Absorption with Organic Chromophores, J. Am. Chem. Soc. 132, 7840 (2010).

[51] O. Varnavski, B. Pinsky and T. Goodson, Entangled Photon Excited Fluorescence in Organic Materials: An Ultrafast Coincidence Detector, J. Phys. Chem. Lett. 8, 388 (2017).

[52] R. Roslyak and S. Mukamel, Multidimensional pump-probe spectroscopy with entangled twin-photon states, Phys. Rev. A 79, 063409 (2009).

[53] R. Roslyak, C. A. Marx and S. Mukamel, Nonlinear spectroscopy with entangled photons: Manipulating quantum pathways of matter, Phys. Rev. A 79, 033832 (2009).

[54] M. Richter and S. Mukamel, Ultrafast double-quantum-coherence spectroscopy of excitons with entangled photons, Phys. Rev. A 82, 013820 (2010).

[55] F. Schlawin, K. E. Dorfman, B. P. Fingerhut and S. Mukamel, Manipulation of two-photon-induced fluorescence spectra of chromophore aggregates with entangled photons: A simulation study, Phys. Rev. A 86, 023851 (2012).

[56] F. Schlawin and S. Mukamel, Two-photon spectroscopy of excitons with entangled photons, J. Chem. Phys. 139, 244110 (2013).

[57] K. E. Dorfman, F. Schlawin and S. Mukamel, Stimulated Raman Spectroscopy with Entangled Light: Enhanced Resolution and Pathway Selection, J. Phys. Chem. Lett. 5, 2843-2849 (2014).

[58] C. Cohen-Tannoudji, G. Grynberg and J. Dupont-Roc, Photons and Atoms: Introduction to Quantum Electrodynamics (Wiley-VCH, Weinheim, Germany, 1997).

[59] D. P. Craig and T. Thirunamanchandran, Molecular Quantum Electrodynamics (Dover Publications, Mineola, New York, 1998).

[60] A. Salam, Molecular Quantum Electrodynamics (John Wiley \& Sons, 2010).

[61] H. P. Breuer and F. Petruccione, The Theory of Open Quantum Systems (Oxford University Press, 2002).

[62] V. May and O. Kühn, Charge and Energy Transfer Dynamics in Molecular Systems: A Theoretical Introduction (John Wiley \& Sons, 2000).

[63] D. Hagenmüller, S. De Liberato and C. Ciuti, Ultrastrong coupling between a cavity resonator and the cyclotron transition of a two-dimensional electron gas in the case of an integer filling factor, Phys. Rev. B 81, 235303 (2010).

[64] F. M. D. Pellegrino et al., Theory of integer quantum Hall polaritons in graphene, Phys. Rev. B 89, 165406 (2014).

[65] S. De Liberato, Light-Matter Decoupling in the Deep Strong Coupling Regime: The Breakdown of the Purcell Effect, Phys. Rev. Lett. 112, 016401 (2014).

[66] V. Y. Chernyak, P. Saurabh and S. Mukamel, Non-linear non-local molecular electrodynamics with nanooptical fields, J. Chem. Phys. 143, 164107 (2015).

[67] B. P. Krueger, G. D. Scholes and G. R. Fleming, Calculation of Couplings and Energy-Transfer Pathways between the Pigments of LH2 by the ab Initio Transition Density Cube Method, J. Phys. Chem. B 102, 5378-5386 (1998).

[68] G. D. Scholes, Long-range resonance energy transfer in molecular systems, Ann. Rev. Phys. Chem. 54, 57-87 (2003).

[69] K. A. Forbes, D. S. Bardshaw and D. L. Andrews, Identifying diamagnetic interactions in scattering and nonlinear optics, Phys. Rev. A 94, 033837 (2016).

[70] K. J. Blow, R. Loudon, S. J. Simon and T. J. Shepherd, Continuum fields in quantum optics, Phys. Rev. A 42, 4102-4114 (1990).

[71] R. Loudon, The Quantum Theory of Light, ( Oxford University Press, Oxford, UK, 2000). 
[72] G. S. Agarwal, Quantum Statistical Theories of Spontaneous Emission and their Relation to other Approaches (Springer, Berlin, 1974).

[73] V. N. Shatokhin, M. Walschaers, F. Schlawin and A. Buchleitner, Coherence turned on by incoherent light, arXiv: 1602.07878 (2016).

[74] V. I. Novoderezhkin and R. van Grondelle, Modeling of excitation dynamics in photosynthetic lightharvesting complexes: exact versus perturbative approaches, J. Phys. B 50, 124003 (2017).

[75] K. Bennett and S. Mukamel, Cascading and local-field effects in non-linear optics revisited: A quantumfield picture based on exchange of photons, J. Chem. Phys. 140, 044313 (2014).

[76] R. de J. Léon-Montiel, Z. Hu and J. Yuen-Zhou, Genuinely quantum effects in nonlinear spectroscopy: vacuum fluctuations and their induced superradiance, arXiv:1606.05717 (2016).

[77] S. Mukamel, Communication: The origin of many-particle signals in nonlinear optical spectroscopy of non-interacting particles, J. Chem. Phys. 145, 041102 (2016).

[78] Z.-Z. Li, L. Bruder, F. Stienkemeier and A. Eisfeld, Probing weak dipole-dipole interaction using phasemodulated non-linear spectroscopy, arXiv:1702.07785 (2017).

[79] D. L. Andrews and T. Thirunamachandran, On three-dimensional rotational averages, J. Chem. Phys. 67, 5026-5033 (1977).

[80] D. Abramavicius et al., Coherent Multidimensional Optical Spectroscopy of Excitons in Molecular Aggregates; Quasiparticle versus Supermolecule Perspectives, Chem. Rev. 109, 2350 - 2408 (2009).

[81] A. M. Branczyk, D. B. Turner and G. D. Scholes, Crossing disciplines - A view on two-dimensional optical spectroscopy, Annalen der Physik 526, 1521-3889 (2014).

[82] N. Christensson, H. F. Kauffmann, T. Pullerits and T. Mančal, Origin of Long-Lived Coherences in LightHarvesting Complexes, J. Phys. Chem. B 116, 7449-7454 (2012).

[83] A. W. Chin et al., Vibrational structures and long-lived electronic coherences, Nature Phys. 9, 113 (2013).

[84] A. Christ et al., Probing multimode squeezing with correlation functions, New J. Phys. 13, 033027 (2011).

[85] K. E. Dorfman and S. Mukamel, Nonlinear spectroscopy with time- and frequency-gated photon counting: A superoperator diagrammatic approach, Phys. Rev. A 86, 013810 (2012).

[86] E. del Valle et al., Theory of Frequency-Filtered and Time-Resolved N-Photon Correlations, Phys. Rev. Lett. 109, 183601 (2012).

[87] U. Harbola and S. Mukamel, Superoperator nonequilibrium Green's function theory of many-body systems; applications to charge transfer and transport in open junctions, Phys. Rep. 465, 191 - 222 (2008).

[88] S. Rahav and S. Mukamel, Chapter 6 - Ultrafast Nonlinear Optical Signals Viewed from the Molecule's Perspective: Kramers-Heisenberg Transition-Amplitudes versus Susceptibilities, Adv. At. Mol. Opt. Phys. 59, 223 - 263 (2010).

[89] K. Bennett and S. Mukamel, Matter and field spectral densities for multidimensional optical response, Chem. Phys. 481, 54-59 (2016).

[90] R. Glenn, K. Bennett,, K. E. Dorfman and S. Mukamel, Photon-exchange induces optical nonlinearities in harmonic systems, J. Phys. B 46, 065401 (2015).

[91] S. Mukamel and K. E. Dorfman, Nonlinear fluctuations and dissipation in matter revealed by quantum light, Phys. Rev. A 91, 053844 (2015).

[92] D. Abramavicius et al., Coherent Multidimensional Optical Spectroscopy of Excitons in Molecular Aggregates; Quasiparticle versus Supermolecule Perspectives, Chem. Rev. 109, 2350-2408 (2009).

[93] F. Schlawin, Quantum-Enhanced Nonlinear Spectroscopy, (Springer Theses, Springer International Publishing, 2016)

[94] S. Mukamel, Principles of nonlinear optical spectroscopy, (Oxford University Press, Oxford, UK, 1999).

[95] D. Nicoletti and A. Cavalleri, Nonlinear light-matter interaction at terahertzfrequencies, Adv. Opt. Photon. 8, 401-464 (2016).

[96] F. Schlawin, K. E. Dorfman and S. Mukamel, Pump-probe spectroscopy using quantum light with twophoton coincidence detection, Phys. Rev. A 93, 023807 (2016).

[97] M. Esposito, U. Harbola and S. Mukamel, Nonequilibrium fluctuations, fluctuation theorems, and counting statistics in quantum systems, Rev. Mod. Phys. 81, 1665-1702 (2009).

[98] H. Walther, B. T. H. Varcoe, B.-G. Englert and T. Becker, Cavity quantum electrodynamics, Rep. Prog. 
Phys. 69, 1325 (2006).

[99] L. Schneebeli, M. Kira and S. W. Koch, Characterization of Strong Light-Matter Coupling in Semiconductor Quantum-Dot Microcavities via Photon-Statistics Spectroscopy, Phys. Rev. Lett. 101, 097401 (2008).

[100] A. Carmele, A. Knorr and M. Richter, Photon statistics as a probe for exciton correlations in coupled nanostructures, Phys. Rev. B 79, 035316 (2009).

[101] M. Aßmann and M. Bayer, Nonlinearity sensing via photon-statistics excitation spectroscopy, Phys. Rev. A 84, 053806 (2011).

[102] T. Kazimierczuk et al., Photon-Statistics Excitation Spectroscopy of a Quantum-Dot Micropillar Laser, Phys. Rev. Lett. 115, 027401 (2015).

[103] M. Strauß et al., Photon-statistics excitation spectroscopy of a single two-level system, Phys. Rev. B 93, 241306 (2016).

[104] J. C. López Carreño et al., Exciting Polaritons with Quantum Light, Phys. Rev. Lett. 115, 196402 (2015).

[105] J. C. López Carreño and F. P. Laussy, Excitation with quantum light. I. Exciting a harmonic oscillator, Phys. Rev. A 94, 063825 (2016).

[106] J. C. López Carreño, C. Sánchez Muñoz, E. Del Valle and F. P. Laussy, Excitation with quantum light. II. Exciting a two-level system, Phys. Rev. A 94, 063826 (2016).

[107] B. R. Mollow, Power Spectrum of Light Scattered by Two-Level Systems, Phys. Rev. 188, 1969-1975 (1969).

[108] M. Kira and S. W. Koch, Quantum-optical spectroscopy of semiconductors, Phys. Rev. Lett. 73, 013813 (2006).

[109] M. Kira and S. W. Koch, Cluster-expansion representation in quantum optics, Phys. Rev. A 78, 022102 (2008).

[110] M. O. Scully and M. S. Zubairy, Quantum Optics, (Cambridge University Press, Cambridge, UK, 1997).

[111] M. Kira et al., Quantum spectroscopy with Schrödinger-cat states, Nature Phys. 7, 799-804 (2011).

[112] M. Mootz et al., Characterizing biexciton coherences with quantum spectroscopy, Phys. Rev. B 89, 155301 (2014).

[113] A. E. Almand-Hunter et al., Quantum droplets of electrons and holes, Nature 506, 471-475 (2014).

[114] L. Mandel and E. Wolf, Optical coherence and quantum Optics (Cambridge University Press, Cambridge, 1995).

[115] A. Christ, B. Brecht, W. Mauerer and C. Silberhorn, Theory of quantum frequency conversion and type-II parametric down-conversion in the high-gain regime, New J. Phys. 15, 053038 (2013).

[116] C. K. Law, I.A. Walmsley and J. H. Eberly, Continuous Frequency Entanglement: Effective Finite Hilbert Space and Entropy Control, Phys. Rev. Lett. 84, 5304-5307 (2000).

[117] W. P. Grice, A. B. U'Ren and I. A. Walmsley, Eliminating frequency and space-time correlations in multiphoton states, Phys. Rev. A 64, 063815 (2001).

[118] C. J. McKinstrie and M. Karlsson, Schmidt decompositions of parametric processes I: Basic theory and simple examples, Opt. Express 21, 1374-1394 (2013).

[119] A. B. U'Ren, K. Banaszek, and I. A. Walmsley, Photon engineering for quantum information processing Quantum Info. Comput. 3, 480 ?502 (2003).

[120] B. Brecht, D. V. Reddy, C. Silberhorn and M. G. Raymer, Photon Temporal Modes: A Complete Framework for Quantum Information Science, Phys. Rev. X 5, 041017 (2015).

[121] F. Schlawin, K. E. Dorfman, B. P. Fingerhut and S. Mukamel, Manipulation of two-photon-induced fluorescence spectra of chromophore aggregates with entangled photons: A simulation study, Phys. Rev. A 86, 23851 (2012).

[122] F. Schlawin, K. E. Dorfman, B. P. Fingerhut and S. Mukamel, Suppression of population transport and control of exciton distributions by entangled photons, Nature Comm. 4, 1782 (2013).

[123] L. J. Salazar, D. A. Guzmán, F. J. Rodríguez and L. Quiroga, Quantum-correlated two-photon transitions to excitons in semiconductor quantum wells, Opt. Express 20, 4470-4483 (2012).

[124] H. Oka, Selective two-photon excitation of a vibronic state by correlated photons, J. Chem. Phys. 134, 124313 (2011). 
[125] H. Oka, Control of vibronic excitation using quantum-correlated photons, J. Chem. Phys. 135, 164304 (2011).

[126] H. Oka, Two-photon process via internal conversion by correlated photon pairs, Phys. Rev. A 85, 013403 (2012).

[127] H. Oka, Highly-efficient entangled two-photon absorption with the assistance of plasmon nanoantenna, J. Phys. B 48, 115503 (2015).

[128] M. G. Raymer, A. H. Marcus, J. R. Widom and D. L. P. Vitullo, Entangled Photon-Pair Two-Dimensional Fluorescence Spectroscopy (EPP-2DFS), J. Phys. Chem. B 117, 15559-15575 (2013).

[129] P. F. Tekavec, G. A. Lott and A. H. Marcus, Fluorescence-detected two-dimensional electronic coherence spectroscopy by acousto-optic phase modulation, J. Chem. Phys. 127, 214307 (2007).

[130] J. Svozolik, J. Peřina Jr. and R. de J. Léon-Montiel, Practical Entangled-Photon Virtual-State Spectroscopy using Intense Twin Beams, arXiv:1608.07326 (2016).

[131] S. Stefanov, On the role of entanglement in two-photon metrology, Quant. Sci. Techn. 2, 025004 (2017).

[132] K. E. Dorfman and S. Mukamel, Multidimensional spectroscopy with entangled light: loop vs ladder delay scanning protocols, New J. Phys. 16, 033013 (2014).

[133] K. E. Dorfman and S. Mukamel, Time-and-frequency-gated photon coincidence counting; a novel multidimensional spectroscopy tool, Phys. Scr. 91, 083004 (2016).

[134] H. Oka, Real-time analysis of two-photon excitation by correlated photons: Pulse-width dependence of excitation efficiency, Phys. Rev. A 81, 053837 (2010).

[135] H. Oka, Efficient selective two-photon excitation by tailored quantum-correlated photons, Phys. Rev. A 81, 063819 (2010).

[136] F. Schlawin and A. Buchleitner, Theory of coherent control with quantum light, New J. Phys. 19, 013009 (2017).

[137] A. González-Tudela, E. del Valle and F. P. Laussy, Optimization of photon correlations by frequency filtering, Phys. Rev. A 91, 043807 (2015).

[138] C. Bernhard, D. Bessire, T. Feurer and A. Stefanov, Shaping frequency-entangled qudits, Phys. Rev. A 88, 032322 (2013).

[139] B. Bessire, C. Bernhard, T. Feurer and A. Stefanov, Versatile shaper-assisted discretization of energytime entangled photons, New J. Phys. 16, 033017 (2014).

[140] A. Stefanov and S. Lerch, Observing the transition from quantum to classical energy correlations with photon pairs, Quant. Inf. Meas, QT6A.16 (2017).

[141] P. Munkhbaatar and K. Myung-Whun, Selection of stimulated Raman scattering signal by entangled photons, Opt. Comm. 383, 581-585 (2017).

[142] F. Schlawin and S. Mukamel, Photon statistics of intense entangled photon pulses, J. Phys. B 46, 175502 (2013).

[143] S. Mukamel and K. E. Dorfman, Nonlinear fluctuations and dissipation in matter revealed by quantum light, Phys. Rev. A 91, 053844 (2015).

[144] M. Kira, S. W. Koch and S. T. Cundiff, Comment on "Nonlinear fluctuations and dissipation in matter revealed by quantum light”, Phys. Rev. A 92, 057801 (2015).

[145] A. A. Kalachev et al.,Biphoton spectroscopy of YAG:Er 3+ crystal, Laser Phys. Lett. 4, 722 (2007).

[146] A. A. Kalachev et al., Biphoton spectroscopy in a strongly nondegenerate regime of SPDC, Laser Phys. Lett. 5, 600 (2008).

[147] D. A. Kalashnikov, Z. Pan, A. I. Kuznetsov and L. A. Krivitsky, Quantum Spectroscopy of Plasmonic Nanostructures, Phys. Rev. X 4, 011049 (2014).

[148] D. A. Kalashnikov et al., Time-resolved spectroscopy with entangled photons, arXiv: 1611.02415 (2016).

[149] N. M. Phan, M. F. Cheng, D. A. Bessarab and L. A. Krivitsky, Interaction of Fixed Number of Photons with Retinal Rod Cells, Phys. Rev. Lett. 112, 213601 (2014).

[150] P. Hamm and M. T. Zanni, Concepts and methods of 2D infrared spectroscopy, (Cambridge University Press, New York, 2011). 